\title{
Correlation and Path Coefficient Analysis in Vegetable Amaranth (Amaranthus tricolor L.) Genotypes
}

\author{
N. Tejaswini ${ }^{*}$, K. Ravinder Reddy ${ }^{1}$, P. Saidaiah ${ }^{2}$ and T. Ramesh ${ }^{3}$ \\ ${ }^{1}$ Department of Horticulture, Vegetable Science, ${ }^{2}$ Department of Genetics and Plant Breeding, \\ SKLTSHU, Hyderabad-500030, India \\ ${ }^{3}$ Department of Crop Physiology, PJTSAU, Rajendranagar, Hyderabad-500030, India \\ *Corresponding author
}

\begin{tabular}{|c|c|}
\hline & A B S T R A C T \\
\hline & In the present study, correlation and path coefficient analysis with 19 yield and yield \\
\hline Keywords & $\begin{array}{l}\text { attributing characters was carried out in } 27 \text { genotypes of vegetable amaranth in } \\
\text { Randomized Block Design with } 3 \text { replications at Vegetable Research Farm, College of }\end{array}$ \\
\hline $\begin{array}{l}\text { Genotypic } \\
\text { correlation, }\end{array}$ & $\begin{array}{l}\text { Horticulture, Rajendranagar, SKLTSHU, Hyderabad during Rabi, 2016. Highly significant } \\
\text { and positive correlation with foliage yield was observed with plant height at } 30 \text { and } 60\end{array}$ \\
\hline $\begin{array}{l}\text { Vegetable, } \\
\text { Amaranth, Yield } \\
\text { and quality } \\
\text {.attributes. }\end{array}$ & $\begin{array}{l}\text { DAS, stem diameter at } 60 \text { and } 90 \text { DAS, stem weight per plant, leaf length, leaf width, leaf } \\
\text { area index, leaf weight per plant and leaf stem ratio at } 30,60 \text { and } 90 \text { DAS, total } \\
\text { chlorophyll content, protein content, ascorbic acid, moisture content and folic acid, } \\
\text { whereas number of branches at } 30,60 \text { and } 90 \text { DAS and number of leaves at } 60 \text { and } 90\end{array}$ \\
\hline Article Info & DAS showed negative association with foliage yield. Path coefficient analysis revealed \\
\hline $\begin{array}{l}\text { Accepted: } \\
29 \text { May } 2017 \\
\text { Available Online: } \\
\text { 10 June } 2017\end{array}$ & $\begin{array}{l}\text { protein content had high to moderate positive direct effect on foliage yield per plant and } \\
\text { these traits recorded significant, positive correlation with foliage yield per plant. It clearly } \\
\text { indicates that direct selection based on these characters would be effective for an increase } \\
\text { in foliage yield. }\end{array}$ \\
\hline
\end{tabular}

\section{Introduction}

The genus, Amaranthus, a member of Amaranthaceae, includes a complex array of wild, weedy and domesticated species and consists of approximately 60 species. In India, the principal species grown for grain purpose are Amaranthus hypochondriacus, A. cruentus, A. caudatus and species grown for vegetable purpose are A. tricolor, A. dubius, A. lividus, A. spinosus and occasionally A.viridus and A.hybridus (Rana et al., 2005). They are tall, soft-wooded annuals, extensively grown throughout India for their green leaves and succulent stem (Aruna et al.,
2009). In the last 20 years amaranthus has been rediscovered as a promising food crop mainly due to its resistance to heat, drought, diseases and pests, and the high nutritional value of both seeds and leaves (National Research Council, 1984). It contains high amount of protein, dietary fiber, dietary minerals and antioxidant compounds like ascorbic acid and betacarotenoid. It has been rated equal or superior in taste to spinach and is considerably higher in protein (14 - 30\% on dry weight basis), minerals (Fe, $\mathrm{Mn}$ and $\mathrm{Zn}$ ) and antioxidants like betacarotenoids (90 - 
$200 \mathrm{mg} / \mathrm{kg}$ ) and ascorbic acid (about 28 $\mathrm{mg} / 100 \mathrm{~g}$ ) compared to any other leafy vegetables (Sarker et al.,). Amaranthus, being a cross-pollinated crop, exhibits wide genetic variability (Arivazhagan and Mohideen 2006). Thus it offers a considerable scope to identify suitable type for any particular region.

In the genetic improvement programme of a crop, association between traits following crosses reflects gene linkages and helps the breeder to decide specific combinations of traits from two parents. The selection for one trait invariably affects a number of associated characters. The estimation of correlation coefficients is of greater value to determine the extent and nature of relationship. Knowledge of such inter-relationship among the characters is useful to the breeders for improving efficiency of selection. In this study, an attempt was made to study the association of characters between each other and their influence on yield.

Importance of correlation study in selection programme is appreciable when highly heritable characters are associated with the important characters like yield. Moreover for important of complex characters like yield per plant direct selection is not much effective, since number of specific forces are involved in the expression of the yield potential of a genotype. Yield is a polygenically controlled complex character affected by a large number of components. Hence, the knowledge of correlation between yield and component characters and among component characters themselves is essential for a rational and direct improvement in yield. The correlation along with path analysis would give a better appreciation of cause and effect relationship between pairs of characters. In the present study, the association of different yield characters and the interrelationships with one another was also investigated.

\section{Materials and Methods}

The investigation was carried out at vegetable research farm of college of horticulture, Department of vegetable science, SKLTSHU, Rajendranagar, Hyderabad, Telangana, India, during February-2016. Twenty seven genotypes of vegetable amaranth were utilized for the study in randomized block design with three replications. Observations were recorded on five randomly selected plants per replication for plant height, stem diameter, stem weight per plant, number of branch per plant, leaf length, leaf width, leaf area index, number of leaves per plant, leaf weight per plant and leaf/stem ratio were recorded at 30,60 and 90 days after sowing, total foliage yield per plant, total chlorophyll content, carotenoids, protein content, ascorbic acid, moisture content, oxalate content, iron content and folic acid. Genotypic and phenotypic correlation coefficients were worked out using the formulae suggested by Al-Jibourie et al., (1958) and path analysis was carried out as suggested by Dewey and $\mathrm{Lu}$ (1959) so as to study the direct and indirect contribution of component characters on foliage yield of vegetable amaranth (Amaranthus tricolor L.) genotypes.

\section{Results and Discussion}

In any crop improvement programme, knowledge on the association of characters is of significant importance since it contributes indirectly to the success of selection. Yield is considered to be a dependent variable on several sub components. In such cases, knowledge of the nature of association between such characters is a great asset for plant breeders to formulate their breeding procedures. It has been suggested that yield might be more effectively increased simultaneously improving one or more yield components (Aruna et al., 2009). 


\section{Correlation coefficient analysis}

Genotypic and phenotypic coefficients among 19 yield and yield attributing characters are presented in table 1 . The correlation studies in the present investigation revealed that the foliage yield per plant recorded positive and significant correlation with leaf weight per plant $\left(\mathrm{r}=0.935^{* *}\right.$ at 30DAS, $\mathrm{r}=0.974 * *$ at 60 DAS, $r=0.953^{* *}$ at 90DAS), leaf stem ratio $\left(\mathrm{r}=0.845^{* *}, 0.866^{* *}, 0.871^{* *}\right.$ at 30,60 and 90 DAS $)$, leaf length $(\mathrm{r}=0.774 * *$, $0.823^{* *}, 0.771^{* *}$ at 30,60 and 90 DAS), protein content $\left(\mathrm{r}=0.748^{* *}\right)$, leaf width $(\mathrm{r}=$ $0.591^{* *}, 0.0 .671^{* *}, 0.747^{* *}$ at 30,60 and 90 DAS $)$, leaf area index $\left(\mathrm{r}=0.561^{* *}, 0.680^{* *}\right.$, $0.590^{* *}$ at 30,60 and 90 DAS) and ascorbic acid $(r=0.604 * *)$. The results suggested that any positive increase in these traits will accelerate the yield potential of vegetable amaranth. So, these traits should be paid attention in vegetable amaranth breeding programmes. The positive and significant correlations between yield and yield components in vegetable amaranth were also reported by Shukla and Singh (2002), Aruna et al., (2009), Shukla et al., (2010), Ahammed et al., (2012), Raja et al., (2012), Chattopadhyay et al., (2013), Mahabub et al., (2013), Hasan et al., (2013), Khurana et al., (2013), Dhangrah et al., (2015) and Gerrano et al., (2015). However, there was a nonsignificant negative correlation between yield and number of branches per plant $(r=-0.262$, $0.222,-0.123$ at 30,60 and 90 DAS respectively) and number of leaves per plant $(\mathrm{r}=-0.031,-0.122$ at 60 and 90DAS respectively). Moreover, the relationship between yield and number of leaves per plant at 30 DAS $(r=0.075)$ was found positive but non-significant. The characters viz., carotenoids $(\mathrm{r}=0.189)$, oxalate content $\quad(\mathrm{r}$ $=0.054)$ showed negligible effect on foliage yield. The characters that showed negligible effect on foliage yield may not be taken into consideration in selection.

\section{Path coefficient analysis}

Correlation coefficients indicate only the general association between any two traits without possible causes of such association. Path coefficient analysis presents a better idea of cause and effect relationship among different characters and plays an important role in determining the degree of relationship between the yield and yield components. Therefore, the path coefficient analysis was performed to partition the correlation coefficient into direct and indirect effect of different characters on yield.

The data pertaining to path coefficient analysis are presented in table 2 . The path coefficient analysis based on foliage yield as a dependent variable revealed that leaf weight per plant $(0.935,0.974$, and 0.953 at 30,60 and 90DAS respectively) had the highest positive direct effect on yield followed by leaf/stem ratio $(0.845,0.866,0.871$ at 30,60 and 90DAS), leaf length $(0.774,0.823,0.771$ at 30,60 and 90DAS), protein content $(0.748)$ and ascorbic acid (0.603), leaf width (0.591, $0.671,0.747)$ at 30,60 and 90DAS), total chlorophyll content $(0.587)$, leaf area index $(0.571,0.680,0.590)$ and folic acid (0.575). These traits also had maximum significant and positive correlation with yield, direct selection for these traits should be done to improve yield of vegetable amaranth.

These findings were in conformity with the results of Aruna et al., (2009), Chattopadhyay et al., (2013) for leaf weight per plant, Aktheruzzaman et al., (2013) and Chattopadhyay et al., (2013) for leaf/stem ratio, Hasan et al., (2013) and Chattopadhyay et al., (2013) for leaf length and leaf width, Shukla et al., (2010) and Khurana et al., (2013) for protein content. It was observed that carotenoids (0.189) exhibited positive and relatively low direct effect on foliage yield. 
Table.1 Phenotypic (P) and Genotypic $(\mathrm{G})$ correlation among nineteen characters related to foliage yield and its attributes in 27 genotypes of vegetable amaranth

\begin{tabular}{|c|c|c|c|c|c|c|c|c|c|c|c|c|c|c|c|c|c|}
\hline \multicolumn{2}{|c|}{ Characters } & & \multicolumn{3}{|c|}{ Plant height $(\mathbf{c m})$} & \multicolumn{3}{|c|}{ Stem diameter $(\mathrm{cm})$} & \multicolumn{3}{|c|}{ Stem weight per plant (g) } & \multicolumn{3}{|c|}{$\begin{array}{l}\text { Number of branches per } \\
\text { plant }\end{array}$} & \multicolumn{3}{|c|}{ Leaf length $(\mathrm{cm})$} \\
\hline & & & 30DAS & 60DAS & 90DAS & 30DAS & 60DAS & 90DAS & 30DAS & 60DAS & 90DAS & 30DAS & 60DAS & 90DAS & 30DAS & 60DAS & 90DAS \\
\hline \multirow{6}{*}{ 氬 } & \multirow[t]{2}{*}{30 DAS } & $\mathrm{P}$ & 1.00 & $0.962 * *$ & $0.939^{* *}$ & $0.521 * *$ & $0.498^{* *}$ & $0.453^{* *}$ & $0.479^{* *}$ & $0.404 * *$ & $0.316^{* *}$ & 0.149 & 0.161 & 0.190 & $0.391 * *$ & $0.409 * *$ & $0.228^{*}$ \\
\hline & & $\mathrm{G}$ & 1.00 & $0.964 * *$ & $0.943 * *$ & $0.525 * *$ & $0.499 * *$ & $0.454 * *$ & $0.485^{* *}$ & $0.406 * *$ & $0.318^{* * *}$ & 0.153 & 0.163 & 0.192 & $0.396^{* * *}$ & $0.422 * *$ & $0.231 *$ \\
\hline & \multirow[t]{2}{*}{60 DAS } & $\mathrm{P}$ & & 1.00 & $0.962 * *$ & $0.542 * *$ & $0.478 * *$ & $0.446^{* *}$ & $0.472^{* *}$ & $0.408 * *$ & $0.282^{*}$ & 0.143 & 0.161 & 0.202 & $0.326^{* * *}$ & $0.357 * *$ & 0.178 \\
\hline & & $\mathrm{G}$ & & 1.00 & $0.963^{* *}$ & $0.546^{* *}$ & $0.481 * *$ & $0.448 * *$ & $0.477 * *$ & $0.409 * *$ & $0.283^{*}$ & 0.144 & 0.164 & 0.205 & $0.329 * *$ & $0.365^{* *}$ & 0.181 \\
\hline & \multirow[t]{2}{*}{90 DAS } & $\mathrm{P}$ & & & 1.00 & $0.533 * *$ & $0.489 * *$ & $0.463 * *$ & $0.558^{* * *}$ & $0.457 * *$ & $0.352 * *$ & 0.207 & $0.230^{*}$ & $0.259 *$ & $0.317 * *$ & $0.344 * *$ & 0.189 \\
\hline & & G & & & 1.00 & $0.538 * *$ & $0.492 * *$ & $0.464 * *$ & $0.564 * *$ & $0.459 * *$ & $0.354 * *$ & 0.209 & $0.233^{*}$ & $0.263 *$ & $0.320 * *$ & $0.353^{* *}$ & 0.191 \\
\hline \multirow{6}{*}{ 离总总 } & \multirow[t]{2}{*}{30 DAS } & $\mathrm{P}$ & & & & 1.00 & $0.933^{* * *}$ & $0.928 * *$ & $0.600^{* * *}$ & $0.458 * *$ & 0.139 & 0.049 & 0.170 & 0.131 & $0.334 * *$ & $0.276^{*}$ & $0.335 * *$ \\
\hline & & $\mathrm{G}$ & & & & 1.00 & $0.941 * *$ & $0.934 * *$ & $0.611 * *$ & $0.462 * *$ & 0.142 & 0.051 & 0.171 & 0.133 & $0.342 * *$ & $0.283^{*}$ & $0.340 * *$ \\
\hline & \multirow[t]{2}{*}{ 60 DAS } & $\mathrm{P}$ & & & & & 1.00 & $0.935 * *$ & $0.609 * *$ & $0.462 * *$ & 0.178 & -0.008 & 0.109 & 0.097 & $0.501 * *$ & $0.393^{* *}$ & 0.479 ** \\
\hline & & $\mathrm{G}$ & & & & & 1.00 & $0.939 * *$ & $0.622 * *$ & $0.467 * *$ & 0.179 & -0.009 & 0.111 & 0.099 & $0.506^{* * *}$ & $0.403^{* *}$ & $0.486^{* * *}$ \\
\hline & \multirow[t]{2}{*}{90 DAS } & $\mathrm{P}$ & & & & & & 1.00 & $0.628^{* * *}$ & $0.489 * *$ & 0.185 & -0.063 & 0.079 & 0.059 & $0.479 * *$ & $0.411 * *$ & $0.468 * *$ \\
\hline & & $\mathrm{G}$ & & & & & & 1.00 & $0.638^{* * *}$ & $0.491 * *$ & 0.186 & -0.066 & 0.081 & 0.061 & $0.485^{* * *}$ & $0.417 * *$ & $0.473 * *$ \\
\hline \multirow{6}{*}{ 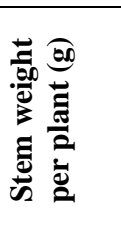 } & \multirow[t]{2}{*}{30 DAS } & $\mathrm{P}$ & & & & & & & 1.00 & $0.871 * *$ & $0.724 * *$ & 0.060 & 0.186 & 0.137 & $0.562 * *$ & $0.582^{* *}$ & $0.499 * *$ \\
\hline & & $\mathrm{G}$ & & & & & & & 1.00 & $0.883 * *$ & $0.732 * *$ & 0.063 & 0.187 & 0.140 & $0.572 * *$ & $0.614^{* *}$ & $0.510 * *$ \\
\hline & \multirow[t]{2}{*}{60 DAS } & $\mathrm{P}$ & & & & & & & & 1.00 & $0.715^{* * *}$ & -0.098 & -0.010 & -0.011 & $0.533^{* * *}$ & $0.629 * *$ & $0.518 * *$ \\
\hline & & $\mathrm{G}$ & & & & & & & & 1.00 & $0.718^{* * *}$ & -0.099 & -0.011 & -0.013 & $0.538^{* * *}$ & $0.643^{* *}$ & $0.525 * *$ \\
\hline & \multirow[t]{2}{*}{90 DAS } & $\mathrm{P}$ & & & & & & & & & 1.00 & 0.169 & $0.249 *$ & 0.192 & $0.539 * *$ & $0.548^{* *}$ & $0.457 * *$ \\
\hline & & $\mathrm{G}$ & & & & & & & & & 1.00 & 0.172 & $0.253^{*}$ & 0.194 & $0.547 * *$ & $0.563^{* *}$ & $0.460 * *$ \\
\hline \multirow{6}{*}{ 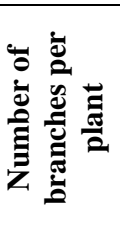 } & \multirow[t]{2}{*}{30 DAS } & $\mathrm{P}$ & & & & & & & & & & 1.00 & $0.951^{* *}$ & $0.914 * *$ & $-0.289 * *$ & $-0.313^{* *}$ & $-0.264^{*}$ \\
\hline & & $\mathrm{G}$ & & & & & & & & & & 1.00 & $0.972^{* * *}$ & $0.938 * *$ & $-0.298 * *$ & $-0.315 * *$ & $-0.269 *$ \\
\hline & \multirow[t]{2}{*}{60 DAS } & $\mathrm{P}$ & & & & & & & & & & & 1.00 & $0.951 * *$ & $-0.248^{*}$ & $-0.258 *$ & -0.182 \\
\hline & & $\mathrm{G}$ & & & & & & & & & & & 1.00 & $0.957 * *$ & $-0.255^{*}$ & $-0.269^{*}$ & -0.187 \\
\hline & \multirow[t]{2}{*}{90 DAS } & $\mathrm{P}$ & & & & & & & & & & & & 1.00 & -0.209 & $\begin{array}{l}-0.208 \\
\end{array}$ & -0.128 \\
\hline & & $\mathrm{G}$ & & & & & & & & & & & & 1.00 & -0.214 & $-0.219^{*}$ & -0.129 \\
\hline \multirow{6}{*}{ 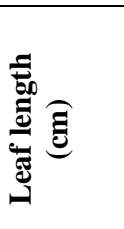 } & \multirow[t]{2}{*}{30 DAS } & $\mathrm{P}$ & & & & & & & & & & & & & 1.00 & $0.863^{* * *}$ & $0.798 * *$ \\
\hline & & $\mathrm{G}$ & & & & & & & & & & & & & 1.00 & $0.899^{* *}$ & $0.815^{* *}$ \\
\hline & 60 DAS & $\mathrm{P}$ & & & & & & & & & & & & & & 1.00 & $0.785 * *$ \\
\hline & & $\mathrm{G}$ & & & & & & & & & & & & & & 1.00 & $0.805 * *$ \\
\hline & 90 DAS & $\mathrm{P}$ & & & & & & & & & & & & & & & 1.00 \\
\hline & & $\mathrm{G}$ & & & & & & & & & & & & & & & 1.00 \\
\hline
\end{tabular}


Table 1. (Contd....)

\begin{tabular}{|c|c|c|c|c|c|c|c|c|c|c|c|c|c|c|c|c|c|}
\hline \multicolumn{2}{|c|}{ Character } & & \multicolumn{3}{|c|}{ Leaf width $(\mathrm{cm})$} & \multicolumn{3}{|c|}{ Leaf area index } & \multicolumn{3}{|c|}{ Number of leaves per plant } & \multicolumn{3}{|c|}{ Leaf weight per plant (g) } & \multicolumn{3}{|c|}{ Leaf /stem ratio } \\
\hline & & & 30DAS & 60DAS & 90DAS & 30DAS & 60DAS & 90DAS & 30DAS & 60DAS & 90DAS & 30DAS & 60DAS & 90DAS & 30DAS & 60DAS & 90DAS \\
\hline \multirow{6}{*}{ 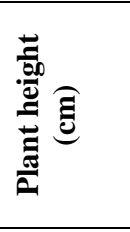 } & \multirow[t]{2}{*}{30 DAS } & $\mathrm{P}$ & $0.455 * *$ & $0.321 * *$ & $0.228 *$ & 0.209 & 0.127 & $0.221 *$ & -0.061 & $-0.233 *$ & -0.199 & $0.389 * *$ & $0.388 * *$ & $0.405 * *$ & $0.301 * *$ & $0.237 *$ & $0.351 * *$ \\
\hline & & $\mathrm{G}$ & $0.476 * *$ & $0.339 * *$ & $0.229 *$ & 0.210 & 0.128 & $0.222 *$ & -0.063 & $-0.234^{*}$ & -0.200 & $0.391 * *$ & $0.390 * *$ & $0.407 * *$ & $0.304 * *$ & $0.239^{*}$ & $0.353 * *$ \\
\hline & \multirow[t]{2}{*}{60 DAS } & $\mathrm{P}$ & $0.439 * *$ & $0.313 * *$ & 0.202 & 0.111 & 0.031 & 0.142 & -0.141 & $-0.298 * *$ & $-0.270 *$ & $0.322 * *$ & $0.351 * *$ & $0.372 * *$ & 0.126 & 0.190 & $0.318 * *$ \\
\hline & & $\mathrm{G}$ & $0.461 * *$ & $0.327 * *$ & 0.205 & 0.112 & 0.032 & 0.143 & -0.143 & $-0.301 * *$ & $-0.272 *$ & $0.323 * *$ & $0.352 * *$ & $0.374 * *$ & 0.127 & 0.192 & $0.321 * *$ \\
\hline & \multirow[t]{2}{*}{90 DAS } & $\mathrm{P}$ & $0.365 * *$ & $0.289 * *$ & 0.171 & 0.199 & 0.085 & 0.209 & -0.039 & $-0.240 *$ & -0.182 & $0.309 * *$ & $0.313 * *$ & $0.356 * *$ & 0.067 & 0.119 & $0.266^{*}$ \\
\hline & & $\mathrm{G}$ & $0.382 * *$ & $0.304 * *$ & 0.172 & 0.203 & 0.086 & 0.210 & -0.040 & $-0.243 *$ & -0.183 & $0.310 * *$ & $0.314 * *$ & $0.359 * *$ & 0.068 & 0.120 & $0.269^{*}$ \\
\hline \multirow{6}{*}{ 离 } & \multirow[t]{2}{*}{30 DAS } & $\mathrm{P}$ & $0.226^{*}$ & $0.215^{*}$ & $0.211 *$ & 0.143 & 0.074 & 0.150 & -0.145 & -0.214 & $-0.254 *$ & $0.289 * *$ & $0.278^{*}$ & $0.304 * *$ & 0.008 & 0.086 & $0.306 * *$ \\
\hline & & $\mathrm{G}$ & $0.252 *$ & $0.222 *$ & $0.215^{*}$ & 0.145 & 0.076 & 0.151 & -0.148 & -0.215 & $-0.257 *$ & $0.290 * *$ & $0.279^{*}$ & $0.306^{* *}$ & 0.009 & 0.089 & $0.315^{* *}$ \\
\hline & \multirow[t]{2}{*}{60 DAS } & $\mathrm{P}$ & $0.299 * *$ & $0.274 *$ & $0.307 * *$ & $0.279 *$ & $0.229^{*}$ & $0.291 * *$ & -0.097 & -0.173 & $-0.221 *$ & $0.427 * *$ & $0.401 * *$ & $0.437 * *$ & 0.155 & $0.226^{*}$ & $0.448 * *$ \\
\hline & & $\mathrm{G}$ & $0.322 * *$ & $0.289 * *$ & $0.309 * *$ & $0.283 *$ & $0.230 *$ & $0.294 * *$ & -0.099 & -0.174 & $-0.223 *$ & $0.431 * *$ & $0.405 * *$ & $0.439 * *$ & 0.159 & $0.227 *$ & $0.455^{* *}$ \\
\hline & \multirow[t]{2}{*}{90 DAS } & $\mathrm{P}$ & $0.229 *$ & $0.265 *$ & $0.318^{* *}$ & $0.352 * *$ & $0.250^{*}$ & $0.275^{*}$ & -0.052 & -0.165 & $-0.231 *$ & $0.475 * *$ & $0.448 * *$ & $0.453 * *$ & $0.221^{*}$ & $0.282 *$ & $0.468 * *$ \\
\hline & & $\mathrm{G}$ & $0.241^{*}$ & $0.274 *$ & $0.319 * *$ & $0.355 * *$ & $0.251 *$ & $0.276^{*}$ & -0.053 & -0.167 & $-0.232 *$ & $0.477 * *$ & $0.450 * *$ & $0.456^{* *}$ & $0.223^{*}$ & $0.282 *$ & $0.471 * *$ \\
\hline \multirow{6}{*}{ 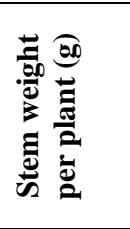 } & \multirow[t]{2}{*}{30 DAS } & $\mathrm{P}$ & $0.479 * *$ & $0.472 * *$ & $0.373 * *$ & $0.429 * *$ & $0.396 * *$ & $0.414 * *$ & 0.033 & -0.186 & -0.171 & $0.466 * *$ & $0.432 * *$ & $0.487 * *$ & 0.003 & 0.046 & 0.149 \\
\hline & & $\mathrm{G}$ & $0.502 * *$ & $0.509 * *$ & $0.383 * *$ & $0.438 * *$ & $0.402 * *$ & $0.419 * *$ & 0.034 & -0.194 & -0.177 & $0.471 * *$ & $0.437 * *$ & $0.493 * *$ & 0.004 & 0.049 & 0.150 \\
\hline & \multirow[t]{2}{*}{60 DAS } & $\mathrm{P}$ & $0.359 * *$ & $0.506 * *$ & $0.400 * *$ & $0.290 * *$ & $0.334 * *$ & $0.322 * *$ & -0.137 & -0.206 & $-0.252 *$ & $0.408 * *$ & $0.473 * *$ & $0.483 * *$ & -0.005 & 0.032 & 0.153 \\
\hline & & $\mathrm{G}$ & $0.378 * *$ & $0.529 * *$ & $0.407 * *$ & $0.293 * *$ & $0.336 * *$ & $0.323 * *$ & -0.138 & -0.211 & $-0.257 *$ & $0.411 * *$ & $0.476 * *$ & $0.487 * *$ & -0.006 & 0.033 & 0.154 \\
\hline & \multirow[t]{2}{*}{90 DAS } & $\mathrm{P}$ & $0.429 * *$ & $0.406 * *$ & $0.339 * *$ & $0.396 * *$ & $0.424 * *$ & $0.457 * *$ & 0.133 & -0.074 & 0.029 & $0.364 * *$ & $0.345 * *$ & $0.416^{* *}$ & 0.019 & 0.034 & -0.085 \\
\hline & & $\mathrm{G}$ & $0.448 * *$ & $0.426^{* *}$ & $0.341 * *$ & $0.399 * *$ & $0.425 * *$ & $0.458 * *$ & 0.135 & -0.075 & 0.030 & $0.365 * *$ & $0.346 * *$ & $0.418 * *$ & 0.020 & 0.035 & -0.086 \\
\hline \multirow{6}{*}{ 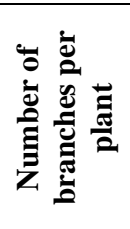 } & \multirow[t]{2}{*}{30 DAS } & $\mathrm{P}$ & -0.043 & -0.115 & -0.016 & $0.238 *$ & 0.149 & $0.294 * *$ & $0.571 * *$ & $0.567 * *$ & $0.656^{* *}$ & -0.134 & $-0.220 *$ & -0.121 & $-0.224 *$ & $-0.223 *$ & $-0.271 *$ \\
\hline & & $\mathrm{G}$ & -0.055 & -0.116 & -0.019 & $0.242 *$ & 0.150 & $0.298 * *$ & $0.580 * *$ & $0.574 * *$ & $0.666^{* *}$ & -0.135 & $-0.224 *$ & -0.124 & $-0.229 *$ & $-0.234 *$ & $-0.279 *$ \\
\hline & \multirow[t]{2}{*}{60 DAS } & $\mathrm{P}$ & 0.006 & -0.019 & 0.074 & $0.292 * *$ & 0.186 & $0.319 * *$ & $0.575 * *$ & $0.518 * *$ & $0.599 * *$ & -0.077 & -0.167 & -0.076 & $-0.215^{*}$ & -0.203 & $-0.258 *$ \\
\hline & & $\mathrm{G}$ & 0.008 & -0.023 & 0.075 & $0.295 * *$ & 0.188 & $0.323 * *$ & $0.592 * *$ & $0.531 * *$ & $0.609 * *$ & -0.078 & -0.171 & -0.079 & $-0.218 *$ & -0.208 & $-0.262 *$ \\
\hline & \multirow[t]{2}{*}{90 DAS } & $\mathrm{P}$ & -0.044 & -0.036 & -0.144 & $0.349 * *$ & $0.266^{*}$ & $0.400 * *$ & $0.614 * *$ & $0.583 * *$ & $0.620 * *$ & 0.010 & -0.059 & 0.011 & -0.099 & -0.090 & -0.139 \\
\hline & & $\mathrm{G}$ & -0.049 & -0.038 & -0.149 & $0.354 * *$ & $0.269 *$ & $0.404 * *$ & $0.630 * *$ & $0.598 * *$ & $0.628 * *$ & 0.011 & -0.060 & 0.012 & -0.103 & -0.092 & -0.143 \\
\hline \multirow{6}{*}{ 䒿 } & \multirow[t]{2}{*}{30 DAS } & $\mathrm{P}$ & $0.637 * *$ & $0.474 * *$ & $0.464 * *$ & $0.504 * *$ & $0.615 * *$ & $0.571 * *$ & -0.098 & $-0.236^{*}$ & $-0.232 *$ & $0.796 * *$ & $0.791 * *$ & $0.814 * *$ & $0.585 * *$ & $0.624 * *$ & $0.621 * *$ \\
\hline & & $\mathrm{G}$ & $0.669 * *$ & $0.495 * *$ & $0.473 * *$ & $0.516^{* *}$ & $0.623 * *$ & $0.579 * *$ & -0.096 & $-0.242 *$ & $-0.240 *$ & $0.806^{* *}$ & $0.801 * *$ & $0.828^{* *}$ & $0.599 * *$ & $0.636^{* *}$ & $0.637 * *$ \\
\hline & 60 DAS & $\mathrm{P}$ & $0.646 * *$ & $0.675 * *$ & $0.586 * *$ & $0.504 * *$ & $0.649 * *$ & $0.565 * *$ & -0.119 & -0.211 & $-0.253 *$ & $0.777 * *$ & $0.826^{* *}$ & $0.807 * *$ & $0.571 * *$ & $0.628 * *$ & $0.596 * *$ \\
\hline & & $\mathrm{G}$ & $0.707 * *$ & $0.708 * *$ & $0.609 * *$ & $0.519 * *$ & $0.664 * *$ & $0.577 * *$ & -0.200 & -0.218 & $-0.254 *$ & $0.796 * *$ & $0.848 * *$ & $0.831 * *$ & $0.588 * *$ & $0.639 * *$ & $0.618 * *$ \\
\hline & 90 DAS & $\mathrm{P}$ & $0.413 * *$ & $0.412 * *$ & $0.499 * *$ & $0.428 * *$ & $0.581 * *$ & $0.505 * *$ & -0.041 & -0.124 & -0.202 & $0.709 * *$ & $0.774 * *$ & $0.765 * *$ & $0.495 * *$ & $0.567 * *$ & $0.574 * *$ \\
\hline & & $\mathrm{G}$ & $0.437 * *$ & $0.443 * *$ & $0.508 * *$ & $0.435 * *$ & $0.586 * *$ & $0.510 * *$ & -0.043 & -0.122 & -0.204 & $0.717 * *$ & $0.781 * *$ & $0.773 * *$ & $0.511 * *$ & $0.579 * *$ & $0.588 * *$ \\
\hline
\end{tabular}


Table 1.(Contd.....)

\begin{tabular}{|c|c|c|c|c|c|c|c|c|c|c|c|}
\hline \multicolumn{2}{|c|}{ Character } & & TCC (mg/g) & $\begin{array}{c}\text { CRT } \\
(\mathrm{mg} / \mathrm{g})\end{array}$ & $\begin{array}{c}\text { PRC } \\
(\mathrm{g} / \mathbf{1 0 0 g})\end{array}$ & $\begin{array}{c}\mathbf{A A} \\
(\mathbf{m g} / \mathbf{1 0 0 g})\end{array}$ & $\operatorname{MC}(\%)$ & $\begin{array}{c}\text { OC } \\
(\mathrm{mg} / \mathbf{1 0 0 g})\end{array}$ & $\begin{array}{c}\text { IC } \\
(\mathrm{mg} / \mathbf{1 0 0 g})\end{array}$ & $\begin{array}{c}\text { FA } \\
(\mathrm{mg} / \mathbf{1 0 0 g})\end{array}$ & TFYP $(\mathrm{g})$ \\
\hline \multirow{3}{*}{ 苛 } & \multirow[t]{2}{*}{30 DAS } & $\mathrm{P}$ & -0.109 & 0.123 & 0.213 & 0.081 & $0.238^{*}$ & 0.189 & -0.008 & 0.086 & $0.365^{* *}$ \\
\hline & & $\mathrm{G}$ & -0.110 & 0.124 & 0.214 & 0.082 & $0.242 *$ & 0.190 & -0.009 & 0.088 & $0.366 * *$ \\
\hline & \multirow[t]{2}{*}{60 DAS } & $\mathrm{P}$ & -0.188 & 0.128 & 0.118 & 0.048 & 0.208 & $0.295 * *$ & -0.083 & -0.019 & $0.333 * *$ \\
\hline \multirow{3}{*}{$\stackrel{\vec{\Xi}}{\stackrel{\Xi}{\Xi}}$} & & $\mathrm{G}$ & -0.189 & 0.129 & 0.119 & 0.049 & 0.209 & $0.296 * *$ & -0.084 & -0.020 & $0.334 * *$ \\
\hline & \multirow[t]{2}{*}{90 DAS } & $\mathrm{P}$ & -0.110 & 0.058 & 0.098 & 0.002 & $0.249^{*}$ & $0.263 *$ & -0.052 & -0.086 & $0.289 * *$ \\
\hline & & $\mathrm{G}$ & -0.111 & 0.059 & 0.099 & 0.003 & $0.250^{*}$ & $0.264^{*}$ & -0.053 & -0.085 & $0.290 * *$ \\
\hline \multirow{6}{*}{ 总 } & \multirow[t]{2}{*}{30 DAS } & $\mathrm{P}$ & -0.045 & -0.116 & 0.154 & -0.071 & 0.154 & $0.271^{*}$ & 0.138 & 0.013 & $0.261^{*}$ \\
\hline & & $\mathrm{G}$ & -0.046 & -0.117 & 0.155 & -0.072 & 0.156 & $0.273^{*}$ & 0.139 & 0.017 & $0.263^{*}$ \\
\hline & \multirow[t]{2}{*}{60 DAS } & $\mathrm{P}$ & 0.123 & -0.126 & $0.253^{*}$ & 0.101 & $0.286 * *$ & 0.072 & $0.266^{*}$ & 0.159 & $0.386 * *$ \\
\hline & & $\mathrm{G}$ & 0.124 & -0.128 & $0.254^{*}$ & 0.102 & $0.289 * *$ & 0.073 & $0.268^{*}$ & 0.162 & $0.387 * *$ \\
\hline & \multirow[t]{2}{*}{90 DAS } & $\mathrm{P}$ & 0.178 & -0.117 & 0.319 ** & 0.107 & $0.284 * * *$ & 0.091 & $0.313^{* *}$ & 0.116 & $0.430 * *$ \\
\hline & & $\mathrm{G}$ & 0.179 & -0.118 & $0.320 * *$ & 0.108 & $0.286 * *$ & 0.092 & $0.314 * *$ & 0.117 & $0.431 * *$ \\
\hline \multirow{6}{*}{ 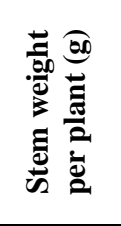 } & \multirow[t]{2}{*}{30 DAS } & $\mathrm{P}$ & 0.187 & 0.132 & 0.208 & 0.149 & $0.361 * *$ & 0.037 & 0.087 & 0.138 & $0.393 * *$ \\
\hline & & $\mathrm{G}$ & 0.189 & 0.137 & 0.209 & 0.151 & $0.371 * *$ & 0.038 & 0.088 & 0.140 & $0.397 * *$ \\
\hline & \multirow[t]{2}{*}{60 DAS } & $\mathrm{P}$ & 0.175 & 0.187 & 0.201 & $0.245^{*}$ & $0.252^{*}$ & 0.108 & 0.108 & 0.108 & $0.435 * *$ \\
\hline & & $\mathrm{G}$ & 0.176 & 0.188 & 0.202 & $0.246^{*}$ & $0.255^{*}$ & 0.109 & 0.109 & 0.109 & $0.436^{* *}$ \\
\hline & \multirow[t]{2}{*}{90 DAS } & $\mathrm{P}$ & $0.301 * *$ & 0.181 & 0.193 & 0.175 & 0.186 & -0.122 & 0.123 & $0.267 *$ & $0.310 * *$ \\
\hline & & $\mathrm{G}$ & $0.302 * *$ & 0.183 & 0.194 & 0.176 & 0.187 & -0.123 & 0.128 & $0.270^{*}$ & $0.311 * *$ \\
\hline \multirow{6}{*}{ 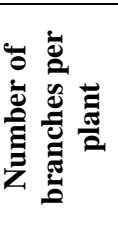 } & \multirow[t]{2}{*}{30 DAS } & $\mathrm{P}$ & $-0.263^{*}$ & $0.252 *$ & -0.064 & $-0.458 * *$ & $0.217 *$ & 0.040 & -0.118 & -0.016 & $-0.260 *$ \\
\hline & & $\mathrm{G}$ & $-0.265^{*}$ & $0.258^{*}$ & -0.065 & $-0.463 * *$ & $0.221 *$ & 0.040 & -0.119 & -0.017 & $-0.262 *$ \\
\hline & \multirow[t]{2}{*}{60 DAS } & $\mathrm{P}$ & $-0.242 *$ & $0.301 * *$ & -0.079 & $-0.411 * *$ & $0.222 *$ & 0.077 & -0.092 & 0.007 & $-0.220 *$ \\
\hline & & $\mathrm{G}$ & $-0.243 *$ & $0.306^{* *}$ & -0.081 & $-0.416 * *$ & $0.227^{*}$ & 0.078 & -0.093 & 0.008 & $-0.222 *$ \\
\hline & \multirow[t]{2}{*}{90 DAS } & $\mathrm{P}$ & -0.202 & $0.351 * *$ & 0.015 & $-0.302 * *$ & $0.330 * *$ & 0.105 & -0.040 & 0.055 & -0.121 \\
\hline & & $\mathrm{G}$ & -0.204 & $0.355^{* *}$ & 0.016 & $-0.305 * *$ & $0.337 * *$ & 0.107 & -0.042 & 0.056 & -0.123 \\
\hline \multirow{6}{*}{ 苞 } & \multirow[t]{2}{*}{30 DAS } & $\mathrm{P}$ & $0.571 * *$ & 0.123 & $0.606 * *$ & $0.480 * *$ & $0.389 * *$ & $-0.263^{*}$ & $0.410 * *$ & $0.510^{* *}$ & $0.766^{* *}$ \\
\hline & & $\mathrm{G}$ & $0.577 * *$ & 0.126 & $0.614 * *$ & $0.484 * *$ & $0.396 * *$ & $-0.265 *$ & $0.415 * *$ & $0.526 * *$ & $0.774 * *$ \\
\hline & \multirow[t]{2}{*}{60 DAS } & $\mathrm{P}$ & $0.567 * *$ & 0.111 & $0.618 * *$ & $0.435 * *$ & $0.359 * *$ & -0.066 & $0.317 * *$ & $0.489 * *$ & $0.804 * *$ \\
\hline & & $\mathrm{G}$ & $0.583 * *$ & 0.112 & $0.633^{* *}$ & $0.446 * *$ & $0.364 * *$ & -0.067 & $0.323^{* *}$ & $0.496 * *$ & $0.823^{* *}$ \\
\hline & \multirow[t]{2}{*}{90 DAS } & $\mathrm{P}$ & $0.549 * *$ & 0.161 & $0.511 * *$ & $0.428 * *$ & $0.241 *$ & 0.006 & $0.305 * *$ & $0.455 * *$ & $0.763 * *$ \\
\hline & & $\mathrm{G}$ & $0.557 * *$ & 0.162 & $0.517 * *$ & $0.433 * *$ & $0.245^{*}$ & 0.006 & $0.309 * *$ & $0.462 * *$ & $0.771 * *$ \\
\hline
\end{tabular}

$\mathrm{TFYP}=$ total foliage yield per plant, $\mathrm{TCC}=$ total chlorophyll content, $\mathrm{CRT}=$ carotenoids, $\mathrm{PRC}=$ protein content, $\mathrm{AA}=\mathrm{ascorbic}$ acid, $\mathrm{MC}=\mathrm{moisture}$ content, $\mathrm{OC}=\mathrm{oxalate}$ content, $\mathrm{IC}=$ iron content, $\mathrm{FA}=$ folic acid 
Table 1. (Contd.....)

\begin{tabular}{|c|c|c|c|c|c|c|c|c|c|c|c|c|c|c|c|c|c|}
\hline \multicolumn{2}{|l|}{ Character } & & \multicolumn{3}{|c|}{ Leaf width (cm) } & \multicolumn{3}{|c|}{ Leaf area index } & \multicolumn{3}{|c|}{ Number of leaves per plant } & \multicolumn{3}{|c|}{ Leaf weight per plant (g) } & \multicolumn{3}{|c|}{ Leaf/stem ratio } \\
\hline & & & 30DAS & 60DAS & 90DAS & 30DAS & 60DAS & 90DAS & 30DAS & 60DAS & 90DAS & 30DAS & 60DAS & 90DAS & 30DAS & 60DAS & 90DAS \\
\hline \multirow{6}{*}{ 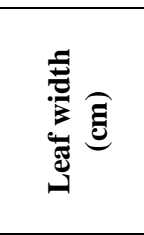 } & \multirow[t]{2}{*}{30 DAS } & $\mathrm{P}$ & 1.00 & $0.655 * *$ & $0.539 * *$ & $0.377 * *$ & $0.492 * *$ & $0.525 * *$ & 0.031 & -0.162 & -0.103 & $0.631 * *$ & $0.611 * *$ & $0.645^{* *}$ & $0.446^{* *}$ & $0.514 * *$ & $0.465^{* *}$ \\
\hline & & $\mathrm{G}$ & 1.00 & $0.725^{* *}$ & $0.564 * *$ & $0.398 * *$ & $0.519 * *$ & $0.549 * *$ & 0.032 & -0.173 & -0.114 & $0.660 * *$ & $0.640 * *$ & $0.673 * *$ & $0.484 * *$ & $0.537 * *$ & $0.493 * *$ \\
\hline & \multirow[t]{2}{*}{60 DAS } & $\mathrm{P}$ & & 1.00 & $0.799 * *$ & $0.459 * *$ & $0.575 * *$ & $0.528 * *$ & 0.069 & -0.011 & -0.036 & $0.627 * *$ & $0.666^{* *}$ & $0.651 * *$ & $0.467 * *$ & $0.499 * *$ & $0.480 * *$ \\
\hline & & $\mathrm{G}$ & & 1.00 & $0.851 * *$ & $0.485 * *$ & $0.599 * *$ & $0.550 * *$ & 0.076 & -0.066 & -0.035 & $0.658 * *$ & $0.699 * *$ & $0.687 * *$ & $0.493 * *$ & $0.533 * *$ & $0.515 * *$ \\
\hline & \multirow[t]{2}{*}{90 DAS } & $\mathrm{P}$ & & & 1.00 & $0.449 * *$ & $0.615^{* *}$ & $0.596^{* *}$ & 0.210 & 0.119 & 0.128 & $0.690 * *$ & $0.711 * *$ & $0.745^{* *}$ & $0.586^{* *}$ & $0.588^{* *}$ & $0.595^{* * *}$ \\
\hline & & $\mathrm{G}$ & & & 1.00 & $0.562 * *$ & $0.623 * *$ & $0.605 * *$ & 0.212 & 0.129 & 0.133 & $0.698 * *$ & $0.721 * *$ & $0.757 * *$ & $0.598 * *$ & $0.598 * *$ & $0.609 * *$ \\
\hline \multirow{6}{*}{ 蔍 } & \multirow[t]{2}{*}{30 DAS } & $\mathrm{P}$ & & & & 1.00 & $0.899 * *$ & $0.889 * *$ & $0.698 * *$ & $0.552 * *$ & $0.537 * *$ & $0.757 * *$ & $0.616^{* *}$ & $0.653 * *$ & $0.594 * *$ & $0.536^{* *}$ & $0.474 * *$ \\
\hline & & $\mathrm{G}$ & & & & 1.00 & $0.908 * *$ & $0.896 * *$ & $0.706 * *$ & $0.561 * *$ & $0.540 * *$ & $0.764 * *$ & $0.618 * *$ & $0.656 * *$ & $0.599 * *$ & $0.542 * *$ & $0.477 * *$ \\
\hline & \multirow[t]{2}{*}{60 DAS } & $\mathrm{P}$ & & & & & 1.00 & $0.935^{* *}$ & $0.550 * *$ & $0.508^{* *}$ & $0.454 * *$ & $0.815^{* *}$ & $0.741 * *$ & $0.767 * *$ & $0.669 * *$ & $0.652 * *$ & $0.544 * *$ \\
\hline & & $\mathrm{G}$ & & & & & 1.00 & $0.938 * *$ & $0.556 * *$ & $0.515^{* *}$ & $0.468 * *$ & $0.818 * *$ & $0.743 * *$ & $0.769 * *$ & $0.677^{* *} *$ & $0.659 * *$ & $0.552 * *$ \\
\hline & \multirow[t]{2}{*}{90 DAS } & $\mathrm{P}$ & & & & & & 1.00 & $0.582 * *$ & $0.510^{* *}$ & $0.575^{* *} *$ & $0.749 * *$ & $0.654 * *$ & $0.734 * *$ & $0.559 * *$ & $0.545 * *$ & 0.492 *** \\
\hline & & $\mathrm{G}$ & & & & & & 1.00 & $0.590 * *$ & $0.517 * *$ & $0.579 * *$ & $0.750 * *$ & $0.656 * *$ & $0.736 * *$ & $0.566 * *$ & $0.549 * *$ & $0.498^{* * *}$ \\
\hline \multirow{6}{*}{ 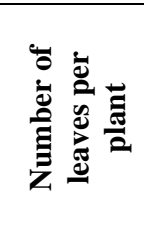 } & \multirow[t]{2}{*}{30 DAS } & $\mathrm{P}$ & & & & & & & 1.00 & $0.852 * *$ & $0.834 * *$ & $0.285 * *$ & 0.104 & 0.167 & $0.247 *$ & 0.149 & 0.047 \\
\hline & & $\mathrm{G}$ & & & & & & & 1.00 & $0.867 * *$ & $0.846^{* *}$ & $0.289 * *$ & 0.106 & 0.168 & $0.252 *$ & 0.153 & 0.048 \\
\hline & \multirow[t]{2}{*}{ 60DAS } & $\mathrm{P}$ & & & & & & & & 1.00 & $0.889 * *$ & 0.121 & 0.007 & 0.138 & 0.164 & 0.075 & -0.007 \\
\hline & & $\mathrm{G}$ & & & & & & & & 1.00 & $0.899 * *$ & 0.124 & 0.008 & 0.139 & 0.165 & 0.077 & -0.009 \\
\hline & \multirow[t]{2}{*}{90 DAS } & $\mathrm{P}$ & & & & & & & & & 1.00 & 0.057 & -0.093 & 0.021 & 0.069 & -0.024 & -0.092 \\
\hline & & $\mathrm{G}$ & & & & & & & & & 1.00 & 0.058 & -0.095 & 0.023 & 0.071 & -0.023 & -0.094 \\
\hline \multirow{6}{*}{ 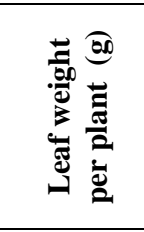 } & \multirow[t]{2}{*}{30 DAS } & $\mathrm{P}$ & & & & & & & & & & 1.00 & $0.952 * *$ & $0.942 * *$ & $0.860 * *$ & $0.853 * *$ & $0.823 * *$ \\
\hline & & $\mathrm{G}$ & & & & & & & & & & 1.00 & $0.955^{* *}$ & $0.947 * *$ & $0.868 * *$ & $0.862 * *$ & $0.834 * *$ \\
\hline & \multirow[t]{2}{*}{60 DAS } & $\mathrm{P}$ & & & & & & & & & & & 1.00 & $0.959 * *$ & $0.843^{* *}$ & $0.878 * *$ & $0.859 * *$ \\
\hline & & $\mathrm{G}$ & & & & & & & & & & & 1.00 & $0.963 * *$ & $0.852 * *$ & $0.887 * *$ & $0.869 * *$ \\
\hline & \multirow[t]{2}{*}{90 DAS } & $\mathrm{P}$ & & & & & & & & & & & & 1.00 & $0.782 * *$ & $0.817 * *$ & $0.840^{* * *}$ \\
\hline & & $\mathrm{G}$ & & & & & & & & & & & & 1.00 & $0.793 * *$ & $0.824 * *$ & 0.849 ** \\
\hline \multirow{6}{*}{ 武 } & \multirow[t]{2}{*}{30 DAS } & $\mathrm{P}$ & & & & & & & & & & & & & 1.00 & $0.962 * *$ & $0.859 * *$ \\
\hline & & $\mathrm{G}$ & & & & & & & & & & & & & 1.00 & $0.980 * *$ & $0.874 * *$ \\
\hline & 60 DAS & $\mathrm{P}$ & & & & & & & & & & & & & & 1.00 & $0.886^{* * *}$ \\
\hline & & $\mathrm{G}$ & & & & & & & & & & & & & & 1.00 & $0.900 * *$ \\
\hline & 90 DAS & $\mathrm{P}$ & & & & & & & & & & & & & & & 1.00 \\
\hline & & $\mathrm{G}$ & & & & & & & & & & & & & & & 1.00 \\
\hline
\end{tabular}


Table 1. (Contd.....)

\begin{tabular}{|c|c|c|c|c|c|c|c|c|c|c|c|}
\hline \multicolumn{2}{|c|}{ Character } & & TCC (mg/g) & CRT (mg/g) & PRC (g/100g) & $\mathrm{AA}(\mathbf{m g} / \mathbf{1 0 0 g})$ & $\operatorname{MC~(\% )~}$ & OC (mg/100g) & IC (mg/100g) & FA $(\mathrm{mg} / \mathbf{1 0 0 g})$ & $\operatorname{TFYP}(\mathrm{g})$ \\
\hline \multirow{5}{*}{ 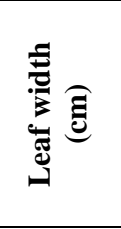 } & \multirow{2}{*}{30 DAS } & $\mathrm{P}$ & 0.121 & $0.402 * *$ & $0.360 * *$ & $0.370 * *$ & $0.357 * *$ & -0.005 & 0.098 & $0.499 * *$ & $0.563 * *$ \\
\hline & & $\mathrm{G}$ & 0.128 & $0.423 * *$ & $0.377 * *$ & $0.390 * *$ & $0.378 * *$ & -0.006 & 0.104 & $0.530 * *$ & $0.591 * *$ \\
\hline & 60 DAS & $\mathrm{G}$ & $0.380 * *$ & 0.164 & $0.482 *$ & $0.429 * *$ & $0.431 * *$ & 0.196 & $0.224 *$ & $0.556 * *$ & $0.671 * *$ \\
\hline & \multirow{2}{*}{90 DAS } & $\mathrm{P}$ & $0.537 * *$ & 0.127 & $0.648 * *$ & $0.399 * *$ & $0.421 * *$ & 0.109 & $0.338 * *$ & $0.605 * *$ & $0.737 * *$ \\
\hline & & $\mathrm{G}$ & $0.544 * *$ & 0.129 & $0.657 * *$ & $0.404 * *$ & $0.429 * *$ & 0.110 & $0.344 * *$ & $0.623 * *$ & $0.747 * *$ \\
\hline \multirow{5}{*}{ 冚 } & 30 DAS & $\mathrm{P}$ & $0.601 * *$ & $0.257^{*}$ & $0.679 * *$ & $0.343 * *$ & $0.762 * *$ & $-0.314 * *$ & $0.349 * *$ & $0.596 * *$ & $0.567 * *$ \\
\hline & \multirow{2}{*}{60 DAS } & $\mathrm{P}$ & $0.626 * *$ & $0.316^{* *}$ & $0.745 * *$ & $0.428 * *$ & $0.678 * *$ & $-0.260 *$ & $0.407 * *$ & $0.693 * *$ & $0.678 * *$ \\
\hline & & $\mathrm{G}$ & $0.628 * *$ & $0.317 * *$ & $0.747 * *$ & $0.430 * *$ & $0.685^{* *}$ & $-0.261 *$ & $0.409 * *$ & $0.697 * *$ & $0.680 * *$ \\
\hline & \multirow{2}{*}{90 DAS } & $\mathrm{P}$ & $0.549 * *$ & $0.267 *$ & $0.674 * *$ & $0.371 * *$ & $0.708 * *$ & -0.208 & $0.424 * *$ & $0.673 * *$ & $0.589 * *$ \\
\hline & & $\mathrm{G}$ & $0.552 * *$ & $0.268 *$ & $0.676 * *$ & $0.372 * *$ & $0.715 * *$ & -0.209 & $0.425 * *$ & $0.679 * *$ & $0.590 * *$ \\
\hline \multirow{4}{*}{ 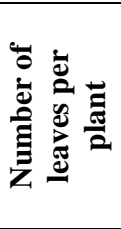 } & \multirow{2}{*}{30 DAS } & $\mathrm{P}$ & $0.219 *$ & $0.358 * *$ & $0.251 *$ & -0.014 & $0.442 * *$ & -0.143 & 0.011 & $0.280 *$ & 0.074 \\
\hline & & $\mathrm{G}$ & $0.221^{*}$ & $0.361 * *$ & $0.255^{*}$ & -0.015 & $0.452 * *$ & -0.145 & 0.012 & $0.283 *$ & 0.075 \\
\hline & \multirow{2}{*}{60 DAS } & $\mathrm{P}$ & 0.131 & $0.277 *$ & $0.265^{*}$ & -0.050 & $0.397 * *$ & -0.147 & 0.080 & $0.290 * *$ & -0.030 \\
\hline & & $\mathrm{G}$ & 0.133 & $0.282^{*}$ & $0.269^{*}$ & -0.051 & $0.404 * *$ & -0.149 & 0.081 & $0.296 * *$ & -0.031 \\
\hline \multirow{6}{*}{ 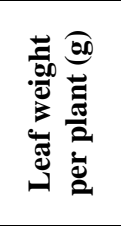 } & \multirow{2}{*}{30 DAS } & $\mathrm{P}$ & $0.582 * *$ & $0.293 * *$ & $0.753 * *$ & $0.584 * *$ & $0.632 * *$ & -0.103 & $0.320 * *$ & $0.655 * *$ & $0.934 * *$ \\
\hline & & $\mathrm{G}$ & $0.584 * *$ & $0.296 * *$ & $0.754 * *$ & $0.585 * *$ & $0.637 * *$ & -0.104 & $0.321 * *$ & $0.661 * *$ & $0.935 * *$ \\
\hline & \multirow{2}{*}{60 DAS } & $\mathrm{P}$ & $0.530 * *$ & $0.243 *$ & $0.705 * *$ & $0.572 * *$ & $0.497 * *$ & 0.006 & $0.324 * *$ & $0.593 * *$ & $0.972 * *$ \\
\hline & & $\mathrm{G}$ & $0.532 * *$ & $0.244^{*}$ & $0.706 * *$ & $0.574 * *$ & $0.501 * *$ & 0.006 & $0.325 * *$ & $0.598 * *$ & $0.974 * *$ \\
\hline & \multirow{2}{*}{90 DAS } & $\mathrm{P}$ & $0.568 * *$ & $0.254^{*}$ & $0.722 * *$ & $0.547 * *$ & $0.502 * *$ & -0.003 & $0.369 * *$ & $0.601 * *$ & $0.950 * *$ \\
\hline & & $\mathrm{G}$ & $0.572 * *$ & $0.255^{*}$ & $0.725 * *$ & $0.550 * *$ & $0.509 * *$ & -0.004 & $0.371 * *$ & $0.607 * *$ & $0.953 * *$ \\
\hline \multirow{6}{*}{ 焉 } & \multirow{2}{*}{30 DAS } & $\mathrm{P}$ & $0.550 * *$ & 0.185 & $0.724 * *$ & $0.537 * *$ & $0.455 * *$ & -0.158 & $0.331 * *$ & $0.610 * *$ & $0.838 * *$ \\
\hline & & $\mathrm{G}$ & $0.553 * *$ & 0.188 & $0.730 * *$ & $0.542 * *$ & $0.458 * *$ & -0.160 & $0.334 * *$ & $0.621 * *$ & $0.845 * *$ \\
\hline & \multirow{2}{*}{60 DAS } & $\mathrm{P}$ & $0.499 * *$ & 0.187 & $0.687 * *$ & $0.503 * *$ & $0.395 * *$ & -0.092 & $0.347 * *$ & $0.591 * *$ & $0.857 * *$ \\
\hline & & $\mathrm{G}$ & $0.506 * *$ & 0.189 & $0.693 * *$ & $0.509 * *$ & $0.401 * *$ & -0.093 & $0.350 * *$ & $0.601 * *$ & $0.866^{* *}$ \\
\hline & \multirow{2}{*}{90 DAS } & $\mathrm{P}$ & $0.420 * *$ & 0.109 & $0.636^{* *}$ & $0.509 * *$ & $0.410 * *$ & 0.023 & $0.351 * *$ & $0.469 * *$ & $0.861 * *$ \\
\hline & & $\mathrm{G}$ & $0.426 * *$ & 0.114 & $0.642 * *$ & $0.515 * *$ & $0.420 * *$ & 0.024 & $0.354 * *$ & $0.477 * *$ & $0.871 * *$ \\
\hline
\end{tabular}

$\mathrm{TFYP}=$ total foliage yield per plant, $\mathrm{TCC}=$ total chlorophyll content, $\mathrm{CRT}=$ carotenoids, $\mathrm{PRC}=$ protein content, $\mathrm{AA}=\mathrm{ascorbic}$ acid, $\mathrm{MC}=$ moisture content, $\mathrm{OC}=\mathrm{oxalate}$ content,

$\mathrm{IC}=$ iron content, $\mathrm{FA}=$ folic acid 
Table 1. (Contd.....)

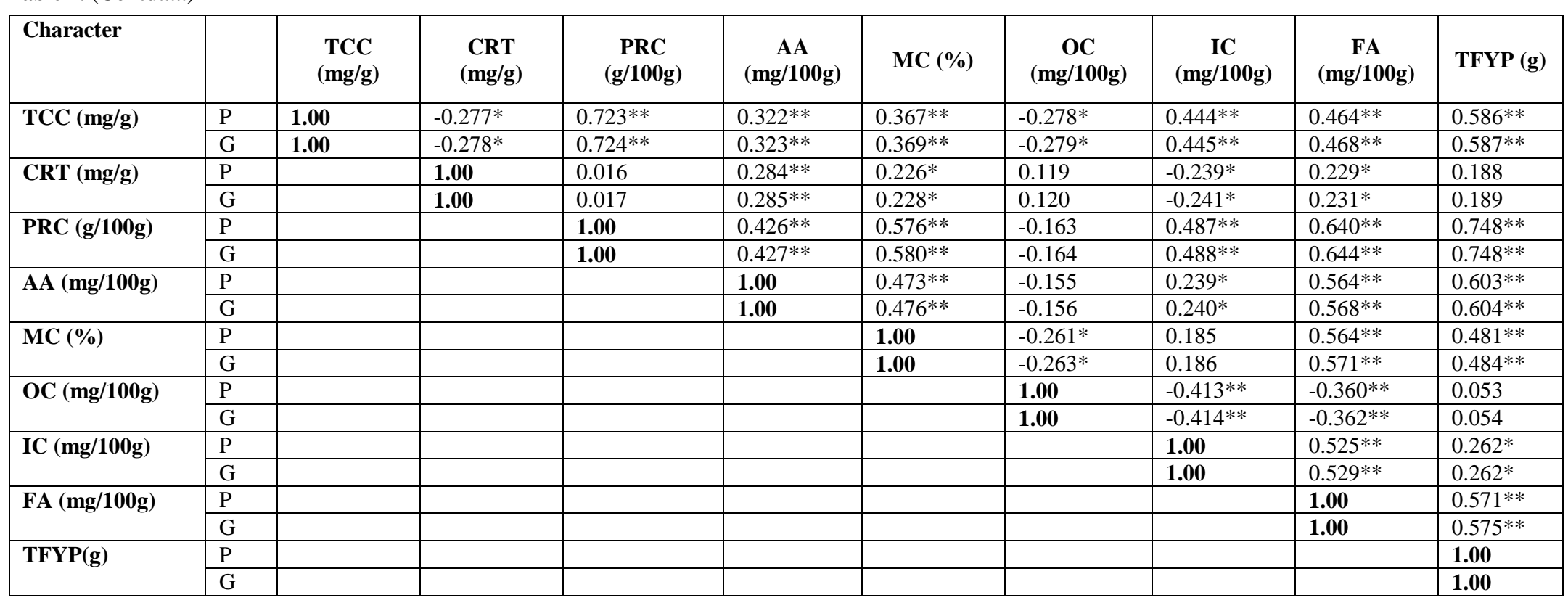

*significant at 5 percent level; **significant at 1 percent level

$\mathrm{TFYP}=$ total foliage yield per plant, $\mathrm{TCC}=$ total chlorophyll content, $\mathrm{CRT}=$ carotenoids, $\mathrm{PRC}=$ protein content, $\mathrm{AA}=$ ascorbic acid, $\mathrm{MC}=\mathrm{moisture}$ content, $\mathrm{OC}=$ oxalate content, $\mathrm{IC}=$ iron content, $\mathrm{FA}=$ folic acid 
Table.2 Phenotypic (P) and Genotypic (G) path coefficient of direct and indirect effects of component characters on foliage yield in 27 genotypes of vegetable amaranth

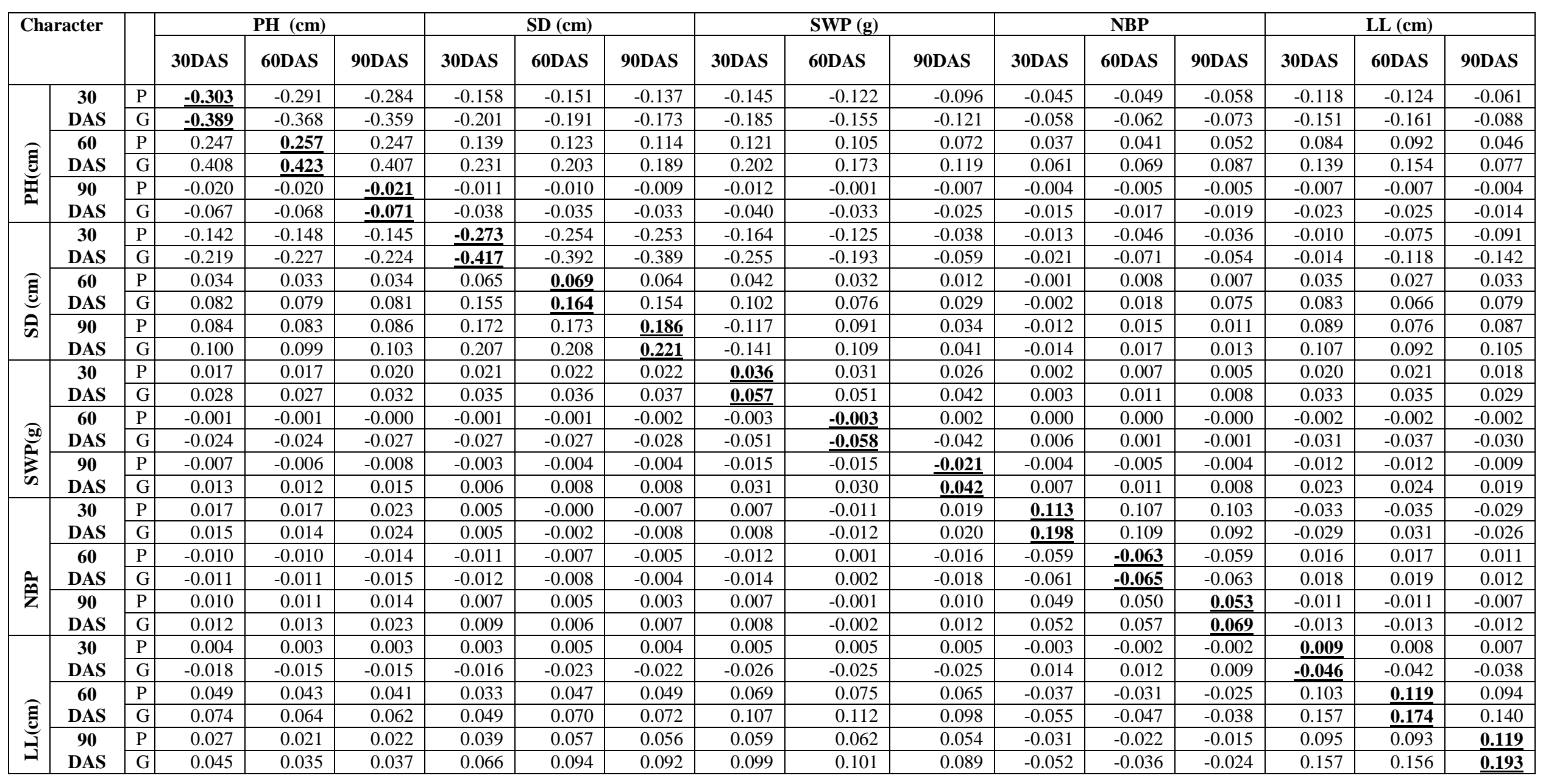

$\mathrm{PH}=$ plant height, $\mathrm{SD}=$ stem diameter, $\mathrm{SWP}=$ stem weight per plant, $\mathrm{NBP}=$ number of branches per plant, $\mathrm{LL}=$ leaf length 
Table 2. (Contd....)

\begin{tabular}{|c|c|c|c|c|c|c|c|c|c|c|c|c|c|c|c|c|c|}
\hline \multirow{2}{*}{\multicolumn{2}{|c|}{ Character }} & & \multirow{2}{*}{\multicolumn{3}{|c|}{ LW (cm) }} & \multirow{2}{*}{\multicolumn{3}{|c|}{ LAI }} & \multirow{2}{*}{\multicolumn{3}{|c|}{ NLP }} & \multirow{2}{*}{\multicolumn{3}{|c|}{ LWP (g) }} & \multirow{2}{*}{\multicolumn{3}{|c|}{ LSR }} \\
\hline & & & & & & & & & & & & & & & & & \\
\hline \multirow{6}{*}{ 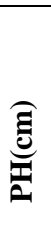 } & \multirow{2}{*}{$\begin{array}{l}\text { 30 } \\
\text { DAS }\end{array}$} & $\mathrm{P}$ & -0.138 & -0.097 & -0.069 & -0.063 & -0.038 & -0.067 & 0.018 & 0.070 & 0.060 & -0.118 & -0.117 & -0.122 & -0.061 & -0.072 & -0.106 \\
\hline & & $\mathrm{G}$ & -0.182 & -0.129 & -0.088 & -0.079 & -0.048 & -0.085 & 0.024 & 0.089 & 0.077 & -0.149 & -0.149 & -0.154 & -0.078 & -0.091 & -0.134 \\
\hline & \multirow{2}{*}{$\begin{array}{l}60 \\
\text { DAS }\end{array}$} & $\mathrm{P}$ & 0.113 & 0.080 & 0.052 & 0.029 & 0.008 & 0.036 & -0.036 & -0.076 & -0.069 & 0.083 & 0.090 & 0.095 & 0.032 & 0.049 & 0.082 \\
\hline & & $\mathrm{G}$ & 0.195 & 0.138 & 0.087 & 0.047 & 0.013 & 0.060 & -0.060 & -0.127 & -0.115 & 0.136 & 0.149 & 0.158 & 0.054 & 0.081 & 0.136 \\
\hline & \multirow{2}{*}{\begin{tabular}{|l|}
90 \\
DAS
\end{tabular}} & $\mathrm{P}$ & 0.008 & 0.006 & 0.004 & 0.004 & 0.002 & 0.004 & -0.001 & -0.005 & -0.004 & 0.007 & 0.007 & 0.008 & 0.001 & 0.003 & 0.006 \\
\hline & & $\mathrm{G}$ & -0.027 & -0.022 & -0.012 & -0.014 & -0.006 & -0.015 & 0.003 & 0.017 & 0.013 & -0.022 & -0.022 & -0.026 & -0.005 & -0.009 & -0.019 \\
\hline \multirow{6}{*}{$\underset{\text { है }}{\stackrel{\Xi}{0}}$} & \multirow{2}{*}{$\begin{array}{l}30 \\
\text { DAS } \\
\end{array}$} & $\mathrm{P}$ & -0.062 & -0.059 & -0.057 & -0.039 & -0.020 & -0.041 & 0.039 & 0.058 & 0.069 & -0.079 & -0.076 & -0.083 & -0.002 & -0.024 & -0.084 \\
\hline & & $\mathrm{G}$ & -0.105 & -0.093 & -0.089 & -0.061 & -0.030 & -0.063 & 0.062 & 0.089 & 0.107 & -0.121 & -0.117 & -0.128 & -0.003 & -0.037 & -0.131 \\
\hline & \multirow{2}{*}{$\begin{array}{l}60 \\
\text { DAS }\end{array}$} & $\mathrm{P}$ & 0.021 & 0.019 & 0.021 & 0.019 & 0.016 & 0.020 & -0.007 & -0.012 & -0.015 & 0.029 & 0.028 & 0.030 & 0.011 & 0.016 & 0.031 \\
\hline & & $\mathrm{G}$ & 0.053 & 0.047 & 0.051 & 0.047 & 0.038 & 0.048 & -0.016 & -0.029 & -0.037 & 0.071 & 0.067 & 0.072 & 0.026 & 0.037 & 0.075 \\
\hline & \multirow{2}{*}{\begin{tabular}{|l|}
90 \\
DAS \\
\end{tabular}} & $\mathrm{P}$ & 0.043 & 0.049 & 0.059 & 0.065 & 0.046 & 0.051 & -0.009 & -0.031 & -0.043 & 0.088 & 0.083 & 0.084 & 0.041 & 0.052 & 0.087 \\
\hline & & $\mathrm{G}$ & 0.053 & 0.061 & 0.071 & 0.079 & 0.056 & 0.061 & -0.012 & -0.037 & -0.051 & 0.106 & 0.099 & 0.101 & 0.049 & 0.062 & 0.105 \\
\hline \multirow{6}{*}{$\overbrace{\infty}^{\infty 00}$} & \multirow{2}{*}{$\begin{array}{l}\text { 30 } \\
\text { DAS }\end{array}$} & $\mathrm{P}$ & 0.017 & 0.017 & 0.013 & 0.015 & 0.014 & 0.015 & 0.001 & -0.007 & -0.006 & 0.016 & 0.015 & 0.017 & 0.000 & 0.002 & 0.005 \\
\hline & & $\mathrm{G}$ & 0.029 & 0.029 & 0.022 & 0.025 & 0.023 & 0.024 & 0.002 & -0.011 & -0.010 & 0.027 & 0.025 & 0.028 & 0.000 & 0.003 & 0.009 \\
\hline & \multirow{2}{*}{$\begin{array}{l}60 \\
\text { DAS }\end{array}$} & $\mathrm{P}$ & -0.001 & -0.002 & -0.001 & -0.001 & -0.001 & -0.001 & -0.000 & -0.001 & 0.001 & -0.001 & -0.002 & -0.002 & -0.000 & -0.000 & -0.001 \\
\hline & & $\mathrm{G}$ & -0.022 & -0.031 & -0.024 & -0.017 & -0.019 & -0.019 & -0.008 & -0.012 & 0.015 & -0.024 & -0.028 & -0.028 & -0.000 & -0.002 & -0.009 \\
\hline & \multirow{2}{*}{$\begin{array}{l}90 \\
\text { DAS }\end{array}$} & $\mathrm{P}$ & -0.009 & -0.009 & -0.007 & -0.009 & -0.009 & -0.009 & -0.003 & 0.002 & -0.001 & -0.008 & -0.007 & -0.009 & -0.000 & -0.001 & 0.002 \\
\hline & & $\mathrm{G}$ & 0.019 & 0.018 & 0.015 & 0.017 & 0.018 & 0.019 & 0.005 & -0.003 & 0.001 & 0.016 & 0.015 & 0.018 & 0.001 & 0.002 & -0.004 \\
\hline \multirow{6}{*}{$\overline{\bar{z}}$} & \multirow{2}{*}{$\begin{array}{l}\text { 30 } \\
\text { DAS }\end{array}$} & $\mathrm{P}$ & -0.005 & -0.013 & -0.002 & 0.027 & 0.017 & 0.033 & 0.064 & 0.064 & 0.074 & -0.015 & -0.025 & -0.014 & -0.025 & -0.025 & -0.031 \\
\hline & & $\mathrm{G}$ & -0.005 & -0.011 & -0.002 & 0.024 & 0.015 & 0.029 & 0.057 & 0.056 & 0.066 & -0.013 & -0.022 & -0.012 & -0.023 & -0.023 & -0.027 \\
\hline & \multirow{2}{*}{$\begin{array}{l}60 \\
\text { DAS }\end{array}$} & $\mathrm{P}$ & -0.000 & 0.001 & 0.005 & -0.018 & -0.012 & -0.020 & -0.036 & -0.033 & -0.038 & 0.005 & 0.001 & 0.005 & 0.014 & 0.013 & 0.016 \\
\hline & & $\mathrm{G}$ & -0.000 & 0.001 & 0.004 & -0.016 & -0.010 & -0.018 & -0.033 & -0.029 & -0.034 & 0.004 & 0.009 & 0.004 & 0.012 & 0.012 & 0.015 \\
\hline & \multirow{2}{*}{$\begin{array}{l}90 \\
\text { DAS }\end{array}$} & $\mathrm{P}$ & 0.002 & 0.002 & 0.008 & 0.019 & 0.014 & 0.021 & 0.033 & 0.031 & 0.033 & 0.001 & -0.003 & 0.001 & -0.005 & -0.005 & -0.007 \\
\hline & & $\mathrm{G}$ & 0.001 & 0.000 & 0.002 & 0.004 & 0.003 & 0.005 & 0.008 & 0.007 & 0.008 & 0.000 & -0.001 & 0.000 & -0.001 & -0.001 & -0.002 \\
\hline \multirow{6}{*}{ 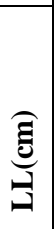 } & \multirow{2}{*}{$\begin{array}{l}\text { 30 } \\
\text { DAS }\end{array}$} & $\mathrm{P}$ & 0.006 & 0.004 & 0.004 & 0.005 & 0.006 & 0.005 & -0.001 & -0.002 & -0.002 & 0.007 & 0.007 & 0.008 & 0.005 & 0.006 & 0.006 \\
\hline & & $\mathrm{G}$ & -0.031 & -0.023 & -0.022 & -0.024 & -0.029 & -0.027 & 0.005 & 0.012 & 0.011 & -0.037 & -0.037 & -0.038 & -0.028 & -0.029 & -0.029 \\
\hline & & $\mathrm{P}$ & 0.077 & 0.081 & 0.069 & 0.060 & 0.077 & 0.067 & -0.014 & -0.025 & -0.030 & 0.093 & 0.099 & 0.096 & 0.069 & 0.075 & 0.071 \\
\hline & DAS & $\mathrm{G}$ & 0.123 & 0.123 & 0.106 & 0.090 & 0.116 & 0.101 & -0.019 & -0.038 & -0.044 & 0.139 & 0.148 & 0.145 & 0.103 & 0.112 & 0.108 \\
\hline & 90 & $\mathrm{P}$ & 0.049 & 0.049 & 0.059 & 0.051 & 0.069 & 0.060 & -0.005 & -0.015 & -0.024 & 0.084 & 0.092 & 0.091 & 0.059 & 0.067 & 0.068 \\
\hline & DAS & $\mathrm{G}$ & 0.085 & 0.086 & 0.098 & 0.084 & 0.113 & 0.099 & -0.008 & -0.024 & -0.039 & 0.139 & 0.151 & 0.149 & 0.099 & 0.112 & 0.114 \\
\hline
\end{tabular}

$\mathrm{PH}=$ plant height, $\mathrm{SD}=$ stem diameter, $\mathrm{SWP}=$ stem weight per plant, $\mathrm{NBP}=$ number of branches per plant, LL=leaf length, $\mathrm{LW}=\mathrm{leaf}$ width, $\mathrm{LAI}=\mathrm{leaf}$ area index, $\mathrm{NLP}=$ number of leaves per plant, LWP=leaf weight per plant, LSR=leaf/stem ratio 
Table 2 (Contd...)

\begin{tabular}{|c|c|c|c|c|c|c|c|c|c|c|c|}
\hline \multicolumn{2}{|c|}{ Character } & & \multirow{2}{*}{$\begin{array}{l}\text { TCC } \\
(\mathbf{m g} / \mathrm{g})\end{array}$} & \multirow{2}{*}{$\begin{aligned} \begin{array}{l}\text { CRT } \\
(\mathrm{mg} / \mathrm{g})\end{array} & \\
& -0.037\end{aligned}$} & \multirow{2}{*}{$\begin{array}{l}\begin{array}{l}\text { PRC } \\
\text { (g/100g) }\end{array} \\
-0.064\end{array}$} & \multirow{2}{*}{$\begin{array}{l}\begin{array}{l}\text { AA } \\
(\mathbf{m g} / \mathbf{1 0 0 g})\end{array} \\
-0.025\end{array}$} & \multirow{2}{*}{$\begin{array}{r}\text { MC (\%) } \\
-0.072\end{array}$} & \multirow{2}{*}{$\begin{array}{r}\begin{array}{l}\text { OC } \\
(\mathbf{m g} / \mathbf{1 0 0 g})\end{array} \\
-0.057\end{array}$} & \multirow{2}{*}{$\begin{array}{r}\begin{array}{l}\text { IC } \\
(\mathbf{m g} / \mathbf{1 0 0 g})\end{array} \\
0.002\end{array}$} & \multirow{2}{*}{$\begin{array}{r}\text { FA }(\mathbf{m g} / \mathbf{1 0 0 g}) \\
-0.026\end{array}$} & \multirow{2}{*}{$\begin{array}{r}\text { TFYP }(\mathbf{g}) \\
0.365^{* *}\end{array}$} \\
\hline \multirow{6}{*}{ 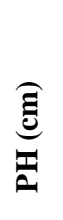 } & \multirow{2}{*}{30 DAS } & $\mathrm{P}$ & & & & & & & & & \\
\hline & & $\mathrm{G}$ & 0.042 & -0.047 & -0.081 & -0.031 & -0.093 & -0.073 & 0.003 & -0.034 & $0.365^{* *}$ \\
\hline & \multirow{2}{*}{60 DAS } & $\mathrm{P}$ & -0.048 & 0.033 & 0.030 & 0.012 & 0.053 & 0.076 & -0.021 & -0.005 & $0.333 * *$ \\
\hline & & $\mathrm{G}$ & -0.079 & 0.054 & 0.049 & 0.020 & 0.089 & 0.125 & -0.035 & -0.008 & $0.334 * *$ \\
\hline & \multirow{2}{*}{90 DAS } & $\mathrm{P}$ & -0.002 & 0.001 & 0.002 & 0.000 & 0.005 & 0.006 & -0.001 & -0.002 & $0.289 * *$ \\
\hline & & $\mathrm{G}$ & 0.008 & -0.004 & -0.007 & -0.000 & -0.018 & -0.019 & 0.004 & 0.006 & $0.289 * *$ \\
\hline \multirow{6}{*}{$\underbrace{\text { है }}_{\text {है }}$} & \multirow{2}{*}{30 DAS } & $\mathrm{P}$ & -0.012 & 0.032 & -0.042 & 0.019 & -0.042 & -0.074 & -0.038 & 0.004 & $0.261^{*}$ \\
\hline & & $\mathrm{G}$ & 0.019 & 0.049 & -0.065 & 0.029 & -0.065 & -0.114 & -0.058 & 0.007 & $0.263^{*}$ \\
\hline & \multirow{2}{*}{60 DAS } & $\mathrm{P}$ & 0.009 & -0.009 & 0.018 & 0.007 & 0.019 & 0.005 & 0.018 & 0.011 & $0.386^{* * *}$ \\
\hline & & $\mathrm{G}$ & 0.020 & -0.021 & 0.042 & 0.017 & 0.048 & 0.012 & 0.044 & 0.027 & $0.387 * *$ \\
\hline & \multirow{2}{*}{90 DAS } & $\mathrm{P}$ & 0.033 & -0.022 & 0.059 & -0.019 & 0.053 & 0.017 & 0.058 & 0.021 & $0.429 * *$ \\
\hline & & $\mathrm{G}$ & 0.039 & -0.026 & 0.071 & 0.024 & 0.063 & 0.020 & 0.069 & 0.026 & $0.431 * *$ \\
\hline \multirow{5}{*}{$\sum_{\infty}^{\infty \infty}$} & \multirow[b]{2}{*}{30 DAS } & $\mathrm{P}$ & 0.007 & 0.005 & 0.007 & 0.005 & 0.013 & 0.001 & 0.003 & 0.005 & $0.393 * *$ \\
\hline & & $\mathrm{G}$ & 0.011 & 0.008 & 0.012 & 0.009 & 0.021 & 0.002 & 0.005 & 0.008 & $0.397 * *$ \\
\hline & \multirow{2}{*}{60 DAS } & $\mathrm{P}$ & -0.001 & -0.000 & -0.001 & -0.001 & -0.001 & -0.000 & -0.000 & -0.000 & $0.435^{* * *}$ \\
\hline & & $\mathrm{G}$ & -0.010 & -0.005 & -0.012 & -0.014 & -0.015 & -0.006 & -0.006 & -0.006 & $0.436 * *$ \\
\hline & 90 DAS & $\mathrm{G}$ & 0.013 & 0.008 & 0.008 & 0.008 & 0.008 & -0.005 & 0.005 & 0.012 & $0.311^{* * *}$ \\
\hline \multirow{6}{*}{ 党 } & \multirow{2}{*}{30 DAS } & $\mathrm{P}$ & -0.029 & 0.028 & -0.007 & 0.052 & 0.024 & -0.005 & -0.013 & -0.002 & $-0.259^{*}$ \\
\hline & & $\mathrm{G}$ & -0.026 & 0.025 & -0.006 & -0.045 & 0.022 & 0.004 & -0.012 & -0.001 & $-0.262^{*}$ \\
\hline & \multirow{2}{*}{60 DAS } & $\mathrm{P}$ & -0.015 & -0.019 & 0.005 & 0.026 & -0.014 & -0.005 & 0.006 & 0.000 & $-0.219 *$ \\
\hline & & $\mathrm{G}$ & 0.014 & -0.017 & 0.005 & 0.023 & -0.013 & -0.004 & -0.005 & -0.000 & $-0.222^{*}$ \\
\hline & \multirow{2}{*}{90 DAS } & $\mathrm{P}$ & -0.011 & 0.019 & 0.001 & -0.016 & 0.018 & 0.006 & -0.002 & 0.003 & -0.121 \\
\hline & & $\mathrm{G}$ & -0.003 & 0.004 & 0.000 & -0.004 & 0.004 & 0.001 & -0.001 & 0.001 & -0.123 \\
\hline \multirow{6}{*}{ 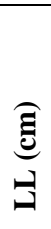 } & \multirow{2}{*}{30 DAS } & $\mathrm{P}$ & 0.005 & 0.001 & 0.006 & 0.004 & 0.004 & -0.002 & 0.004 & 0.005 & 0.766 ** \\
\hline & & $\mathrm{G}$ & -0.027 & -0.006 & -0.028 & -0.022 & -0.018 & 0.012 & -0.019 & -0.024 & $0.774 * *$ \\
\hline & & $\mathrm{P}$ & 0.068 & 0.013 & 0.074 & 0.052 & 0.043 & -0.008 & 0.038 & 0.058 & $0.804 * *$ \\
\hline & 60 DAS & $\mathrm{G}$ & 0.102 & 0.019 & 0.110 & 0.078 & 0.064 & -0.012 & 0.056 & 0.086 & $0.823 * *$ \\
\hline & & $\mathrm{P}$ & 0.065 & 0.019 & 0.061 & 0.051 & 0.029 & 0.001 & 0.036 & 0.054 & $0.763 * *$ \\
\hline & 90 DAS & $\mathrm{G}$ & 0.108 & 0.031 & 0.099 & 0.084 & 0.047 & 0.001 & 0.059 & 0.089 & $0.771 * *$ \\
\hline
\end{tabular}

$\mathrm{PH}=$ plant height, $\mathrm{SD}=$ stem diameter, $\mathrm{SWP}=$ stem weight per plant, $\mathrm{NBP}=$ number of branches per plant, $\mathrm{LL}=$ leaf length, TFYP= total foliage yield per plant, $\mathrm{TCC}=$ total chlorophyll content, $\mathrm{CRT}=$ carotenoids, $\mathrm{PRC}=$ protein content, $\mathrm{AA}=$ ascorbic acid, $\mathrm{MC}=$ moisture content, $\mathrm{OC}=$ oxalate content, $\mathrm{IC}=\mathrm{iron}$ content, $\mathrm{FA}=$ folic acid 
Table 2. (Contd....)

\begin{tabular}{|c|c|c|c|c|c|c|c|c|c|c|c|c|c|c|c|c|c|}
\hline \multirow{2}{*}{\multicolumn{2}{|c|}{ Character }} & & \multicolumn{3}{|c|}{ Plant height $(\mathrm{cm})$} & \multicolumn{3}{|c|}{ Stem diameter $(\mathrm{cm})$} & \multicolumn{3}{|c|}{ Stem weight per plant (g) } & \multicolumn{3}{|c|}{ Number of branches per plant } & \multicolumn{3}{|c|}{ Leaf length $(\mathrm{cm})$} \\
\hline & & & 30DAS & 60DAS & 90DAS & 30DAS & 60DAS & 90DAS & 30DAS & 60DAS & 90DAS & 30DAS & 60DAS & 90DAS & 30DAS & 60DAS & 90DAS \\
\hline \multirow{6}{*}{ 氖 } & \multirow[t]{2}{*}{30 DAS } & $\mathrm{P}$ & -0.017 & -0.016 & -0.014 & -0.009 & -0.012 & -0.009 & -0.018 & -0.014 & -0.016 & 0.002 & -0.000 & -0.002 & -0.024 & -0.025 & -0.016 \\
\hline & & $\mathrm{G}$ & -0.014 & -0.013 & -0.011 & -0.007 & -0.009 & -0.007 & -0.014 & -0.011 & -0.013 & 0.001 & -0.000 & -0.001 & -0.019 & -0.020 & -0.013 \\
\hline & \multirow[t]{2}{*}{60 DAS } & $\mathrm{P}$ & 0.022 & 0.021 & 0.019 & 0.014 & 0.018 & 0.018 & 0.032 & 0.034 & 0.027 & -0.008 & -0.001 & 0.002 & 0.032 & 0.045 & 0.023 \\
\hline & & $\mathrm{G}$ & 0.020 & 0.020 & 0.018 & 0.014 & 0.018 & 0.017 & 0.031 & 0.033 & 0.026 & -0.007 & -0.001 & 0.002 & 0.031 & 0.044 & 0.028 \\
\hline & \multirow[t]{2}{*}{90 DAS } & $\mathrm{P}$ & 0.003 & 0.003 & 0.002 & 0.003 & 0.004 & 0.004 & 0.005 & 0.005 & 0.004 & -0.000 & -0.001 & -0.002 & 0.006 & 0.008 & 0.006 \\
\hline & & $\mathrm{G}$ & 0.007 & 0.006 & 0.005 & 0.006 & 0.009 & 0.009 & 0.011 & 0.012 & 0.001 & -0.001 & 0.002 & 0.004 & 0.013 & 0.017 & 0.014 \\
\hline \multirow{6}{*}{ 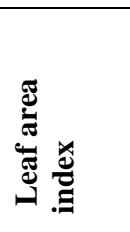 } & \multirow[t]{2}{*}{30 DAS } & $\mathrm{P}$ & -0.010 & -0.005 & -0.009 & -0.007 & -0.013 & -0.017 & -0.021 & -0.014 & -0.019 & -0.016 & -0.014 & -0.017 & -0.024 & -0.024 & -0.021 \\
\hline & & $\mathrm{G}$ & -0.005 & -0.003 & -0.005 & -0.004 & -0.007 & -0.009 & -0.011 & -0.008 & -0.010 & -0.006 & -0.008 & -0.009 & -0.014 & -0.014 & -0.011 \\
\hline & \multirow[t]{2}{*}{60 DAS } & $\mathrm{P}$ & -0.013 & -0.003 & 0.009 & -0.008 & -0.024 & -0.026 & -0.041 & -0.034 & -0.043 & -0.015 & -0.019 & -0.027 & -0.063 & -0.067 & -0.059 \\
\hline & & $\mathrm{G}$ & -0.012 & -0.003 & -0.008 & -0.007 & -0.022 & -0.025 & -0.039 & -0.033 & -0.042 & -0.015 & -0.018 & -0.026 & -0.061 & -0.065 & -0.058 \\
\hline & \multirow[t]{2}{*}{90 DAS } & $\mathrm{P}$ & -0.052 & -0.033 & -0.049 & -0.035 & -0.068 & -0.064 & -0.097 & -0.075 & -0.107 & -0.069 & -0.075 & -0.094 & -0.134 & -0.133 & -0.118 \\
\hline & & $\mathrm{G}$ & -0.067 & -0.043 & -0.064 & -0.046 & -0.089 & -0.084 & -0.127 & -0.098 & -0.139 & -0.091 & -0.098 & -0.123 & -0.176 & -0.175 & -0.155 \\
\hline \multirow{6}{*}{ 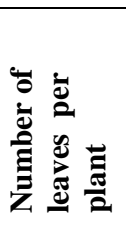 } & \multirow[t]{2}{*}{30 DAS } & $\mathrm{P}$ & 0.001 & 0.001 & 0.001 & 0.001 & 0.001 & 0.001 & -0.000 & 0.001 & -0.001 & -0.005 & -0.005 & -0.005 & 0.001 & 0.001 & 0.000 \\
\hline & & $\mathrm{G}$ & 0.003 & 0.007 & 0.002 & 0.008 & 0.005 & 0.003 & -0.002 & -0.007 & -0.007 & -0.021 & -0.030 & -0.032 & 0.005 & 0.006 & 0.002 \\
\hline & \multirow[t]{2}{*}{60 DAS } & $\mathrm{P}$ & 0.001 & 0.001 & 0.001 & 0.001 & 0.001 & 0.001 & 0.000 & -0.002 & -0.002 & -0.002 & -0.002 & -0.002 & 0.001 & 0.001 & 0.000 \\
\hline & & $\mathrm{G}$ & -0.000 & -0.001 & -0.000 & -0.000 & -0.000 & -0.000 & 0.000 & -0.001 & -0.001 & 0.001 & 0.001 & 0.001 & -0.000 & -0.000 & -0.000 \\
\hline & \multirow[t]{2}{*}{90 DAS } & $\mathrm{P}$ & -0.023 & -0.031 & -0.021 & -0.029 & -0.026 & -0.027 & -0.019 & -0.029 & 0.003 & 0.076 & 0.069 & 0.072 & -0.027 & -0.029 & -0.023 \\
\hline & & $\mathrm{G}$ & -0.042 & -0.058 & -0.039 & -0.054 & -0.047 & -0.049 & -0.037 & -0.054 & 0.006 & 0.141 & 0.129 & 0.133 & -0.051 & -0.054 & -0.043 \\
\hline \multirow{6}{*}{ 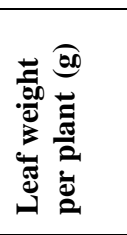 } & \multirow[t]{2}{*}{ 30 DAS } & $\mathrm{P}$ & 0.129 & 0.107 & 0.102 & 0.095 & 0.141 & 0.157 & 0.154 & 0.135 & 0.120 & -0.044 & -0.026 & 0.003 & 0.263 & 0.257 & 0.235 \\
\hline & & $\mathrm{G}$ & 0.161 & 0.133 & 0.127 & 0.119 & 0.177 & 0.197 & 0.194 & 0169 & 0.150 & -0.056 & -0.031 & 0.005 & 0.332 & 0.328 & 0.295 \\
\hline & \multirow[t]{2}{*}{60 DAS } & $\mathrm{P}$ & 0.105 & 0.095 & 0.085 & 0.075 & 0.109 & 0.121 & 0.117 & 0.128 & 0.093 & -0.059 & -0.045 & -0.013 & 0.214 & 0.224 & 0.209 \\
\hline & & $\mathrm{G}$ & 0.077 & 0.061 & 0.062 & 0.055 & 0.080 & 0.089 & 0.087 & 0.094 & 0.069 & -0.044 & -0.034 & -0.012 & 0.159 & 0.168 & 0.155 \\
\hline & \multirow[t]{2}{*}{90 DAS } & $\mathrm{P}$ & 0.121 & 0.111 & 0.106 & 0.091 & 0.130 & 0.135 & 0.145 & 0.144 & 0.124 & -0.036 & -0.023 & 0.003 & 0.243 & 0.241 & 0.228 \\
\hline & & $\mathrm{G}$ & 0.115 & 0.106 & 0.102 & 0.087 & 0.125 & 0.121 & 0.140 & 0.139 & 0.119 & -0.035 & -0.022 & 0.003 & 0.236 & 0.237 & 0.220 \\
\hline \multirow{6}{*}{ 总 } & \multirow[t]{2}{*}{ 30 DAS } & $\mathrm{P}$ & 0.026 & 0.016 & 0.009 & 0.001 & 0.020 & 0.029 & 0.000 & -0.001 & 0.002 & -0.029 & -0.028 & -0.013 & 0.076 & 0.074 & 0.065 \\
\hline & & $\mathrm{G}$ & 0.041 & 0.026 & 0.014 & 0.002 & 0.032 & 0.045 & 0.001 & -0.001 & 0.004 & -0.046 & -0.044 & -0.021 & 0.121 & 0.119 & 0.103 \\
\hline & 60 DAS & $\mathrm{P}$ & -0.028 & -0.022 & -0.014 & -0.010 & -0.026 & -0.033 & -0.005 & -0.004 & -0.004 & 0.026 & 0.024 & 0.011 & -0.073 & -0.073 & -0.066 \\
\hline & & $\mathrm{G}$ & -0.042 & -0.034 & -0.021 & -0.016 & -0.040 & -0.050 & -0.009 & -0.006 & -0.007 & 0.042 & 0.037 & 0.016 & -0.113 & -0.114 & -0.103 \\
\hline & 90 DAS & $\mathrm{P}$ & 0.016 & 0.015 & 0.012 & 0.014 & 0.020 & 0.021 & 0.007 & 0.007 & -0.004 & -0.012 & -0.012 & -0.006 & 0.028 & 0.027 & 0.026 \\
\hline & & $\mathrm{G}$ & 0.020 & 0.019 & 0.016 & 0.018 & 0.026 & 0.027 & 0.009 & 0.009 & -0.005 & -0.016 & -0.015 & -0.008 & 0.037 & 0.036 & 0.034 \\
\hline
\end{tabular}


Table 2. (Contd....)

\begin{tabular}{|c|c|c|c|c|c|c|c|c|c|c|c|c|c|c|c|c|c|}
\hline \multirow{2}{*}{\multicolumn{2}{|c|}{ Character }} & & \multicolumn{3}{|c|}{ Leaf width (cm) } & \multicolumn{3}{|c|}{ Leaf area index } & \multicolumn{3}{|c|}{ Number of leaves per plant } & \multicolumn{3}{|c|}{ Leaf weight per plant (g) } & \multicolumn{3}{|c|}{ Leaf/ stem ratio } \\
\hline & & & 30DAS & 60DAS & 90DAS & 30DAS & 60DAS & 90DAS & 30DAS & 60DAS & 90DAS & 30DAS & 60DAS & 90DAS & 30DAS & 60DAS & 90DAS \\
\hline \multirow{6}{*}{ 䒿 } & \multirow[t]{2}{*}{30 DAS } & $\mathrm{P}$ & $-\mathbf{0 . 0 3 8}$ & -0.025 & -0.021 & -0.014 & -0.019 & -0.020 & -0.001 & 0.006 & 0.004 & -0.024 & -0.023 & -0.025 & -0.017 & -0.030 & -0.018 \\
\hline & & $\mathrm{G}$ & -0.029 & -0.021 & -0.016 & -0.011 & -0.015 & -0.016 & -0.001 & 0.005 & 0.003 & -0.019 & -0.018 & -0.019 & -0.014 & -0.015 & -0.014 \\
\hline & \multirow[t]{2}{*}{60 DAS } & $\mathrm{P}$ & $\overline{0.044}$ & $\underline{0.067}$ & 0.054 & 0.031 & 0.039 & 0.036 & 0.005 & -0.001 & -0.002 & 0.042 & 0.045 & 0.044 & 0.031 & 0.034 & 0.032 \\
\hline & & $\mathrm{G}$ & 0.045 & $\overline{0.062}$ & 0.052 & 0.029 & 0.037 & 0.034 & 0.005 & -0.000 & -0.002 & 0.041 & 0.043 & 0.042 & 0.030 & 0.033 & 0.032 \\
\hline & \multirow[t]{2}{*}{90 DAS } & $\mathrm{P}$ & 0.007 & 0.010 & 0.013 & 0.007 & 0.008 & 0.008 & 0.003 & 0.002 & 0.002 & 0.009 & 0.009 & 0.009 & 0.008 & 0.008 & 0.008 \\
\hline & & $\mathrm{G}$ & 0.016 & 0.024 & 0.028 & 0.016 & 0.018 & 0.017 & 0.006 & 0.004 & 0.004 & 0.019 & 0.020 & 0.021 & 0.017 & 0.017 & 0.017 \\
\hline \multirow{6}{*}{ 导 } & \multirow[t]{2}{*}{30 DAS } & $\mathrm{P}$ & -0.018 & -0.022 & -0.026 & -0.048 & -0.043 & -0.043 & -0.034 & -0.027 & -0.026 & -0.036 & -0.029 & -0.031 & -0.029 & -0.026 & -0.023 \\
\hline & & $\mathrm{G}$ & -0.010 & -0.013 & -0.015 & -0.026 & -0.024 & -0.023 & -0.019 & -0.015 & -0.014 & -0.020 & -0.016 & -0.017 & -0.016 & -0.014 & -0.013 \\
\hline & \multirow[t]{2}{*}{60 DAS } & $\mathrm{P}$ & -0.050 & -0.059 & -0.063 & -0.092 & 0.102 & -0.096 & -0.056 & -0.052 & -0.048 & -0.084 & -0.076 & -0.079 & -0.069 & -0.067 & -0.056 \\
\hline & & $\mathrm{G}$ & -0.051 & -0.059 & -0.061 & -0.089 & $-\overline{0.098}$ & -0.092 & -0.055 & -0.051 & -0.046 & -0.080 & -0.073 & -0.076 & -0.066 & -0.065 & -0.054 \\
\hline & \multirow[t]{2}{*}{90 DAS } & $\mathrm{P}$ & -0.123 & -0.124 & -0.139 & -0.208 & -0.219 & -0.234 & -0.136 & -0.119 & -0.135 & -0.175 & -0.153 & -0.172 & -0.131 & -0.128 & -0.115 \\
\hline & & $\mathrm{G}$ & -0.167 & -0.167 & -0.184 & -0.272 & -0.285 & -0.304 & -0.179 & -0.157 & -0.176 & -0.228 & -0.199 & -0.224 & -0.172 & -0.167 & -0.151 \\
\hline \multirow{6}{*}{ 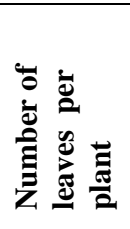 } & \multirow[t]{2}{*}{30 DAS } & $\mathrm{P}$ & -0.000 & -0.001 & -0.002 & -0.006 & -0.004 & -0.006 & -0.008 & -0.007 & -0.007 & -0.002 & -0.001 & -0.001 & -0.002 & -0.001 & -0.000 \\
\hline & & $\mathrm{G}$ & -0.001 & -0.004 & -0.011 & -0.036 & -0.029 & -0.030 & -0.051 & -0045 & -0.043 & -0.015 & -0.005 & -0.009 & -0.013 & -0.008 & -0.002 \\
\hline & \multirow[t]{2}{*}{60 DAS } & $\mathrm{P}$ & -0.001 & -0.000 & -0.000 & -0.002 & -0.001 & -0.001 & $\overline{-0.002}$ & -0.003 & -0.003 & -0.000 & 0.000 & -0.000 & -0.001 & -0.000 & 0.000 \\
\hline & & $\mathrm{G}$ & -0.000 & 0.000 & 0.000 & 0.001 & 0.001 & 0.001 & 0.001 & $\overline{0.002}$ & 0.001 & 0.000 & 0.000 & 0.000 & 0.000 & 0.000 & 0.000 \\
\hline & \multirow[t]{2}{*}{90 DAS } & $\mathrm{P}$ & -0.012 & -0.004 & 0.015 & 0.062 & 0.054 & 0.066 & 0.096 & 0.103 & $\underline{0.115}$ & 0.007 & -0.011 & 0.002 & 0.008 & -0.003 & -0.011 \\
\hline & & $\mathrm{G}$ & -0.024 & -0.007 & 0.028 & 0.114 & 0.099 & 0.123 & 0.179 & 0.190 & $\underline{0.212}$ & 0.012 & -0.020 & 0.004 & 0.015 & -0.005 & -0.019 \\
\hline \multirow{6}{*}{ 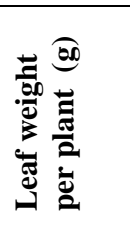 } & \multirow[t]{2}{*}{30 DAS } & $\mathrm{P}$ & 0.209 & 0.208 & 0.229 & 0.250 & 0.269 & 0.248 & 0.094 & 0.040 & $\overline{0.019}$ & $\underline{0.331}$ & 0.315 & 0.312 & 0.285 & 0.282 & 0.273 \\
\hline & & $\mathrm{G}$ & 0.272 & 0.271 & 0.288 & 0.315 & 0.337 & 0.309 & 0.119 & 0.051 & 0.024 & 0.412 & 0.393 & 0.390 & 0.358 & 0.355 & 0.344 \\
\hline & \multirow[t]{2}{*}{60 DAS } & $\mathrm{P}$ & 0.166 & 0.180 & 0.193 & 0.167 & 0.201 & 0.177 & 0.028 & 0.002 & -0.025 & 0.644 & 0.271 & 0.259 & 0.229 & 0.378 & 0.233 \\
\hline & & $\mathrm{G}$ & 0.127 & 0.139 & 0.143 & 0.123 & 0.147 & 0.130 & 0.021 & 0.002 & -0.019 & 0.189 & $\overline{\mathbf{0 . 1 9 8}}$ & 0.191 & 0.169 & 0.176 & 0.172 \\
\hline & \multirow[t]{2}{*}{90 DAS } & $\mathrm{P}$ & 0.192 & 0.194 & 0.222 & 0.195 & 0.229 & 0.219 & 0.049 & 0.011 & 0.006 & 0.281 & $\overline{0.286}$ & 0.298 & 0.233 & 0.243 & 0.250 \\
\hline & & $\mathrm{G}$ & 0.192 & 0.196 & 0.216 & 0.187 & 0.219 & 0.209 & 0.048 & 0.011 & 0.005 & 0.269 & 0.274 & 0.285 & 0.226 & 0.235 & 0.242 \\
\hline \multirow{6}{*}{ 焉 } & \multirow[t]{2}{*}{30 DAS } & $\mathrm{P}$ & 0.058 & 0.061 & 0.076 & 0.077 & 0.087 & 0.073 & 0.032 & 0.021 & 0.009 & 0.112 & 0.109 & 0.102 & 0.130 & 0.125 & 0.112 \\
\hline & & $\mathrm{G}$ & 0.098 & 0.099 & 0.120 & 0.121 & 0.136 & 0.114 & 0.051 & 0.033 & 0.014 & 0.175 & 0171 & 0.159 & 0.202 & 0.198 & 0.176 \\
\hline & 60 DAS & $\mathrm{P}$ & -0.060 & -0.058 & -0.069 & -0.063 & -0.076 & -0.064 & -0.017 & -0.009 & 0.003 & -0.099 & -0.103 & -0.096 & $-\overline{-0.113}$ & -0.117 & -0.104 \\
\hline & & $\mathrm{G}$ & -0.095 & -0.095 & -0.106 & -0.096 & -0.117 & -0.098 & -0.027 & -0.014 & 0.004 & -0.153 & -0.157 & -0.146 & -0.174 & -0.177 & -0.159 \\
\hline & 90 DAS & $\mathrm{P}$ & 0.021 & 0.022 & 0.027 & 0.022 & 0.025 & 0.022 & 0.002 & -0.000 & -0.004 & 0.038 & 0.039 & 0.038 & 0.039 & 0.040 & $\underline{0.046}$ \\
\hline & & $\mathrm{G}$ & 0.029 & 0.029 & 0.035 & 0.028 & 0.032 & 0.029 & 0.003 & -0.000 & -0.005 & 0.048 & 0.050 & 0.049 & 0.051 & 0.052 & $\underline{0.058}$ \\
\hline
\end{tabular}


Table 2. (Contd....)

\begin{tabular}{|c|c|c|c|c|c|c|c|c|c|c|c|}
\hline \multicolumn{2}{|c|}{ Character } & & TCC (mg/g) & CRT (mg/g) & PRC (g/100g) & $\mathrm{AA}(\mathrm{mg} / \mathbf{1 0 0 g})$ & MC (\%) & OC (mg/100g) & IC (mg/100g) & FA (mg/100g) & TFYP (g) \\
\hline \multirow{6}{*}{ 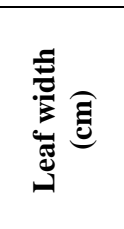 } & \multirow{2}{*}{30 DAS } & $\mathrm{P}$ & -0.005 & -0.015 & -0.014 & -0.014 & -0.014 & 0.000 & -0.004 & -0.019 & $0.563^{* *}$ \\
\hline & & $\mathrm{G}$ & -0.004 & -0.012 & -0.012 & -0.011 & -0.011 & 0.000 & -0.003 & -0.015 & $0.591 * *$ \\
\hline & \multirow{2}{*}{60 DAS } & $\mathrm{P}$ & 0.025 & 0.011 & 0.031 & 0.028 & 0.028 & 0.013 & 0.014 & 0.036 & $0.645^{* *}$ \\
\hline & & $\mathrm{G}$ & 0.023 & 0.010 & 0.029 & 0.026 & 0.027 & 0.012 & 0.014 & 0.034 & $0.671 * *$ \\
\hline & \multirow{2}{*}{90 DAS } & $\mathrm{P}$ & 0.007 & 0.002 & 0.008 & 0.005 & 0.005 & 0.001 & 0.004 & 0.008 & $0.737 * *$ \\
\hline & & $\mathrm{G}$ & 0.015 & 0.004 & 0.019 & 0.011 & 0.012 & 0.003 & 0.009 & 0.018 & $0.747 * *$ \\
\hline \multirow{6}{*}{ 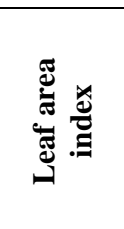 } & \multirow{2}{*}{30 DAS } & $\mathrm{P}$ & -0.029 & -0.012 & -0.033 & -0.017 & -0.037 & 0.015 & -0.017 & -0.029 & $0.567 * *$ \\
\hline & & $\mathrm{G}$ & -0.016 & -0.007 & -0.018 & -0.009 & -0.020 & 0.008 & -0.009 & -0.016 & $0.571^{* *}$ \\
\hline & \multirow{2}{*}{60 DAS } & $P$ & -0.064 & -0.032 & -0.076 & -0.044 & -0.069 & 0.027 & -0.042 & -0.071 & $0.678^{* *}$ \\
\hline & & $\mathrm{G}$ & -0.062 & -0.031 & -0.073 & -0.042 & -0.067 & 0.026 & -0.040 & -0.068 & $0.679 * *$ \\
\hline & \multirow{2}{*}{90 DAS } & $\mathrm{P}$ & -0.129 & -0.063 & -0.158 & -0.087 & -0.166 & 0.049 & -0.099 & -0.158 & $0.589 * *$ \\
\hline & & $\mathrm{G}$ & -0.168 & -0.081 & -0.205 & -0.113 & -0.217 & 0.063 & -0.129 & -0.206 & $0.589 * *$ \\
\hline \multirow{6}{*}{ 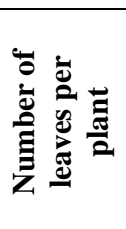 } & \multirow[b]{2}{*}{30 DAS } & $\mathrm{P}$ & -0.002 & -0.003 & -0.002 & 0.000 & -0.004 & 0.001 & -0.000 & -0.002 & 0.074 \\
\hline & & $\mathrm{G}$ & -0.011 & -0.019 & -0.013 & 0.001 & -0.023 & 0.007 & -0.001 & -0.015 & 0.075 \\
\hline & \multirow{2}{*}{60 DAS } & $\mathrm{P}$ & 0.004 & 0.001 & 0.001 & -0.000 & 0.001 & -0.004 & 0.002 & 0.001 & -0.030 \\
\hline & & $\mathrm{G}$ & 0.007 & 0.007 & 0.006 & -0.001 & 0.002 & -0.002 & 0.001 & 0.006 & -0.031 \\
\hline & \multirow{2}{*}{90 DAS } & $\mathrm{P}$ & 0.018 & 0.023 & 0.027 & -0.009 & 0.043 & -0.020 & 0.021 & 0.037 & -0.121 \\
\hline & & $\mathrm{G}$ & 0.034 & 0.043 & 0.050 & -0.017 & 0.079 & -0.038 & 0.039 & 0.069 & -0.122 \\
\hline \multirow{6}{*}{ 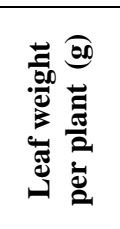 } & \multirow{2}{*}{30 DAS } & $\mathrm{P}$ & 0.193 & 0.097 & 0.249 & 0.193 & 0.209 & -0.034 & 0.106 & 0.217 & $0.934 * *$ \\
\hline & & $\mathrm{G}$ & 0.241 & 0.122 & 0.311 & 0.241 & 0.262 & -0.043 & 0.132 & 0.272 & $0.935^{* *}$ \\
\hline & \multirow{2}{*}{60 DAS } & $\mathrm{P}$ & 0.144 & 0.066 & 0.191 & 0.155 & 0.135 & 0.002 & 0.088 & 0.161 & $0.972^{* *}$ \\
\hline & & $\mathrm{G}$ & 0.106 & 0.048 & 0.140 & 0.114 & 0.099 & 0.001 & 0.065 & 0.119 & $0.974 * *$ \\
\hline & \multirow{2}{*}{90 DAS } & $\mathrm{P}$ & 0.169 & 0.076 & 0.215 & 0.163 & 0.149 & -0.001 & 0.110 & 0.179 & $0.949 * *$ \\
\hline & & $\mathrm{G}$ & 0.163 & 0.073 & 0.206 & 0.157 & 0.145 & -0.001 & 0.106 & 0.173 & $0.953 * *$ \\
\hline \multirow{6}{*}{ 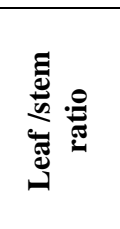 } & \multirow{2}{*}{30 DAS } & $\mathrm{P}$ & 0.072 & 0.024 & 0.094 & 0.070 & 0.059 & -0.021 & 0.043 & 0.079 & $0.838^{* *}$ \\
\hline & & $\mathrm{G}$ & 0.111 & 0.038 & 0.147 & 0.109 & 0.092 & -0.032 & 0.067 & 0.125 & $0.845^{* *}$ \\
\hline & \multirow{2}{*}{60 DAS } & $\mathrm{P}$ & -0.058 & -0.022 & -0.080 & -0.059 & -0.046 & 0.011 & -0.041 & -0.069 & $0.857 * *$ \\
\hline & & $\mathrm{G}$ & -0.089 & -0.034 & -0.123 & -0.090 & -0.071 & 0.017 & -0.062 & -0.107 & $0.866^{* *}$ \\
\hline & \multirow{2}{*}{90 DAS } & $\mathrm{P}$ & 0.019 & 0.005 & 0.029 & 0.023 & 0.019 & 0.001 & 0.016 & 0.021 & $0.861 * *$ \\
\hline & & $\mathrm{G}$ & 0.025 & 0.007 & 0.037 & 0.029 & 0.024 & 0.001 & 0.021 & 0.028 & $0.871^{* *}$ \\
\hline
\end{tabular}

$\mathrm{TFYP}=$ total foliage yield per plant, $\mathrm{TCC}=$ total chlorophyll content, $\mathrm{CRT}=$ carotenoids, $\mathrm{PRC}=$ protein content, $\mathrm{AA}=$ ascorbic acid, $\mathrm{MC}=$ moisture content, $\mathrm{OC}=\mathrm{oxalate}$ content,

$\mathrm{IC}=$ iron content, $\mathrm{FA}=$ folic acid 
Table 2. (Contd....)

\begin{tabular}{|c|c|c|c|c|c|c|c|c|c|c|c|c|c|c|c|c|}
\hline \multicolumn{2}{|l|}{ Character } & \multicolumn{3}{|c|}{ PH (cm) } & \multicolumn{3}{|c|}{ SD (cm) } & \multicolumn{3}{|c|}{ SWP (g) } & \multicolumn{3}{|c|}{ NBP } & \multicolumn{3}{|c|}{ LL (cm) } \\
\hline & & 30DAS & 60DAS & 90DAS & 30DAS & 60DAS & 90DAS & 30DAS & 60DAS & 90DAS & 30DAS & 60DAS & 90DAS & 30DAS & 60DAS & 90DAS \\
\hline \multirow{2}{*}{ TCC (mg/g) } & $\mathrm{P}$ & 0.008 & 0.013 & 0.008 & 0.003 & -0.009 & -0.012 & -0.013 & -0.012 & -0.021 & 0.018 & 0.017 & 0.014 & -0.039 & -0.039 & -0.038 \\
\hline & $\mathrm{G}$ & 0.018 & 0.030 & -0.018 & 0.007 & -0.017 & -0.029 & -0.030 & -0.028 & -0.048 & 0.042 & 0.039 & 0.033 & -0.092 & -0.093 & -0.089 \\
\hline \multirow{2}{*}{ CRT (mg/g) } & $\mathrm{P}$ & -0.014 & -0.015 & -0.007 & 0.013 & 0.015 & 0.014 & -0.015 & -0.010 & -0.021 & -0.029 & -0.035 & -0.041 & -0.014 & -0.013 & -0.019 \\
\hline & $\mathrm{G}$ & -0.018 & -0.019 & -0.009 & 0.017 & 0.018 & 0.017 & -0.011 & -0.013 & -0.026 & -0.037 & -0.044 & -0.051 & -0.018 & -0.016 & -0.023 \\
\hline$P C(g / 100 g)$ & $\mathrm{P}$ & 0.048 & 0.026 & 0.022 & 0.034 & 0.057 & 0.071 & 0.046 & 0.045 & 0.043 & -0.014 & -0.018 & 0.003 & 0.136 & 0.138 & 0.114 \\
\hline \multirow{2}{*}{$\begin{array}{c}\mathbf{A A} \\
(\mathbf{m g} / \mathbf{1 0 0 g})\end{array}$} & $\mathrm{P}$ & 0.008 & 0.005 & 0.000 & -0.007 & 0.010 & 0.011 & 0.015 & 0.025 & 0.018 & -0.047 & -0.042 & -0.031 & 0.049 & 0.045 & 0.044 \\
\hline & $\mathrm{G}$ & 0.006 & 0.003 & 0.000 & -0.005 & 0.007 & 0.008 & 0.011 & 0.017 & 0.013 & -0.033 & -0.021 & -0.022 & 0.034 & 0.032 & 0.031 \\
\hline \multirow{2}{*}{ MC (\%) } & $\mathrm{P}$ & -0.019 & -0.017 & -0.020 & -0.013 & -0.023 & -0.023 & -0.029 & -0.021 & -0.015 & -0.018 & -0.018 & -0.027 & -0.042 & -0.029 & -0.019 \\
\hline & $\mathrm{G}$ & -0.008 & -0.007 & -0.009 & -0.005 & -0.009 & -0.009 & -0.013 & -0.009 & -0.006 & -0.008 & -0.008 & -0.011 & -0.014 & -0.012 & -0.008 \\
\hline \multirow{2}{*}{$\begin{array}{c}\mathrm{OC} \\
(\mathrm{mg} / 100 \mathrm{~g})\end{array}$} & $\mathrm{P}$ & 0.003 & 0.005 & 0.004 & 0.005 & 0.001 & 0.002 & 0.001 & 0.002 & -0.002 & 0.001 & 0.001 & 0.002 & -0.004 & -0.001 & 0.000 \\
\hline & $\mathrm{G}$ & 0.007 & 0.011 & 0.009 & 0.010 & 0.003 & 0.003 & 0.001 & 0.004 & -0.005 & 0.002 & 0.003 & 0.004 & -0.009 & -0.003 & 0.000 \\
\hline \multirow{2}{*}{ IC (mg/100g) } & $\mathrm{P}$ & 0.001 & 0.014 & 0.009 & -0.023 & -0.044 & -0.051 & -0.014 & -0.018 & -0.021 & 0.019 & 0.015 & 0.007 & -0.068 & -0.052 & -0.050 \\
\hline & $\mathrm{G}$ & 0.001 & 0.012 & 0.007 & -0.019 & -0.038 & -0.044 & -0.012 & -0.015 & -0.018 & 0.017 & 0.013 & 0.006 & -0.059 & -0.046 & -0.043 \\
\hline FA (mg/100g) & $\mathrm{P}$ & 0.005 & -0.001 & -0.005 & -0.001 & 0.009 & 0.007 & 0.008 & 0.006 & 0.015 & -0.001 & 0.002 & 0.003 & 0.029 & 0.028 & 0.026 \\
\hline
\end{tabular}

$\mathrm{TFYP}=$ total foliage yield per plant, $\mathrm{TCC}=$ total chlorophyll content, $\mathrm{CRT}=$ carotenoids, $\mathrm{PRC}=$ protein content, $\mathrm{AA}=\mathrm{ascorbic}$ acid, $\mathrm{MC}=$ moisture content, $\mathrm{OC}=\mathrm{oxalate}$ content, IC=iron content, FA=folic acid 
Table 2. (Contd....)

\begin{tabular}{|c|c|c|c|c|c|c|c|c|c|c|c|c|c|c|c|c|}
\hline \multicolumn{2}{|l|}{ Character } & \multicolumn{3}{|c|}{ Leaf width (cm) } & \multicolumn{3}{|c|}{ Leaf area index } & \multicolumn{3}{|c|}{ Number of leaves per plant } & \multicolumn{3}{|c|}{ Leaf weight per plant (g) } & \multicolumn{3}{|c|}{ Leaf/ stem ratio } \\
\hline & & 30DAS & 60DAS & 90DAS & 30DAS & 60DAS & 90DAS & 30DAS & 60DAS & 90DAS & 30DAS & 60DAS & 90DAS & 30DAS & 60DAS & 90DAS \\
\hline \multirow{2}{*}{ TCC (mg/g) } & $\mathrm{P}$ & -0.008 & -0.025 & -0.037 & -0.041 & -0.043 & -0.038 & -0.015 & -0.009 & -0.011 & -0.040 & -0.037 & -0.039 & -0.038 & -0.034 & -0.029 \\
\hline & $\mathrm{G}$ & -0.021 & -0.061 & -0.087 & -0.097 & -0.101 & -0.088 & -0.035 & -0.021 & -0.026 & -0.094 & -0.085 & -0.092 & -0.089 & -0.081 & -0.068 \\
\hline \multirow{2}{*}{ CRT (mg/g) } & $\mathrm{P}$ & -0.046 & -0.019 & -0.015 & -0.029 & -0.037 & -0.031 & -0.041 & -0.032 & -0.023 & -0.034 & -0.028 & -0.029 & -0.021 & -0.022 & -0.013 \\
\hline & $\mathrm{G}$ & -0.061 & -0.024 & -0.019 & -0.037 & -0.046 & -0.039 & -0.052 & -0.041 & -0.029 & -0.043 & -0.035 & -0.037 & -0.027 & -0.027 & -0.016 \\
\hline \multirow{2}{*}{ PC (g/100g) } & $\mathrm{P}$ & 0.040 & 0.103 & 0.145 & 0.152 & 0.166 & 0.151 & 0.056 & 0.059 & 0.053 & 0.168 & 0.158 & 0.161 & 0.162 & 0.154 & 0.142 \\
\hline & $\mathrm{G}$ & 0.089 & 0.114 & 0.155 & 0.161 & 0.176 & 0.159 & 0.060 & 0.063 & 0.056 & 0.178 & 0.166 & 0.171 & 0.172 & 0.163 & 0.151 \\
\hline \multirow{2}{*}{$\begin{array}{c}\mathbf{A A} \\
(\mathbf{m g} / \mathbf{1 0 0 g})\end{array}$} & $\mathrm{P}$ & 0.038 & 0.042 & 0.041 & 0.035 & 0.044 & 0.038 & -0.002 & -0.005 & -0.008 & 0.059 & 0.059 & 0.056 & 0.055 & 0.052 & 0.052 \\
\hline & $\mathrm{G}$ & 0.028 & 0.031 & 0.029 & 0.025 & 0.031 & 0.027 & -0.001 & -0.004 & -0.006 & 0.042 & 0.041 & 0.039 & 0.039 & 0.036 & 0.037 \\
\hline \multirow{2}{*}{$\operatorname{MC}(\%)$} & $\mathrm{P}$ & -0.029 & -0.034 & -0.034 & -0.062 & -0.055 & -0.058 & -0.036 & -0.032 & -0.030 & -0.052 & -0.041 & -0.041 & -0.037 & -0.032 & -0.034 \\
\hline & $\mathrm{G}$ & -0.013 & -0.015 & -0.015 & -0.026 & -0.023 & -0.024 & -0.015 & -0.014 & -0.013 & -0.022 & -0.017 & -0.017 & -0.015 & -0.014 & -0.014 \\
\hline \multirow{2}{*}{$\begin{array}{c}\text { OC } \\
(\mathrm{mg} / 100 \mathrm{~g})\end{array}$} & $\mathrm{P}$ & -0.001 & 0.003 & 0.002 & -0.005 & -0.004 & -0.003 & -0.002 & -0.002 & -0.003 & -0.002 & 0.000 & 0.000 & -0.003 & -0.002 & 0.000 \\
\hline & $\mathrm{G}$ & -0.000 & 0.007 & 0.004 & -0.012 & -0.009 & -0.008 & -0.005 & -0.006 & -0.007 & -0.004 & 0.000 & -0.000 & -0.006 & -0.003 & 0.001 \\
\hline \multirow{2}{*}{ IC (mg/100g) } & $\mathrm{P}$ & -0.016 & -0.035 & -0.056 & -0.058 & -0.067 & -0.069 & -0.002 & -0.013 & -0.030 & -0.053 & -0.053 & -0.061 & -0.055 & -0.057 & -0.058 \\
\hline & $\mathrm{G}$ & -0.015 & -0.032 & -0.049 & -0.049 & -0.058 & -0.060 & -0.002 & -0.011 & -0.026 & -0.046 & -0.046 & -0.053 & -0.047 & -0.049 & -0.050 \\
\hline \multirow{2}{*}{ FA (mg/100g) } & $\mathrm{P}$ & 0.029 & 0.031 & 0.035 & 0.035 & 0.040 & 0.039 & 0.016 & 0.017 & 0.018 & 0.038 & 0.034 & 0.035 & 0.035 & 0.034 & 0.027 \\
\hline & $\mathrm{G}$ & 0.001 & 0.003 & 0.008 & 0.008 & 0.011 & 0.010 & 0.000 & 0.000 & 0.000 & 0.009 & 0.007 & 0.005 & 0.005 & 0.004 & 0.000 \\
\hline
\end{tabular}

$\mathrm{TFY}=$ total foliage yield per plant, $\mathrm{TCC}=$ total chlorophyll content, $\mathrm{CRT}=$ carotenoids, $\mathrm{PRC}=$ protein content, $\mathrm{AA}=\mathrm{ascorbic}$ acid, $\mathrm{MC}=\mathrm{moisture}$ content, $\mathrm{OC}=\mathrm{oxalate}$ content,

$\mathrm{IC}=$ iron content, $\mathrm{FA}=$ folic acid 
Table 2. (Contd....)

\begin{tabular}{|c|c|c|c|c|c|c|c|c|c|c|}
\hline Character & & $\begin{array}{c}\text { TCC } \\
(\mathrm{mg} / \mathrm{g})\end{array}$ & $\begin{array}{c}\text { CRT } \\
(\mathrm{mg} / \mathrm{g})\end{array}$ & $\begin{array}{c}\text { PRC } \\
(\mathrm{g} / \mathbf{1 0 0 g})\end{array}$ & $\underset{(\mathbf{m g} / \mathbf{1 0 0 g})}{\mathbf{A A}}$ & $\begin{array}{l}\text { MC } \\
(\%)\end{array}$ & $\begin{array}{c}\mathrm{OC} \\
(\mathrm{mg} / 100 \mathrm{~g})\end{array}$ & $\underset{(\mathrm{mg} / 100 \mathrm{IC})}{\text {. }}$ & $\begin{array}{c}\text { FA } \\
(\mathbf{m g} / 100 g)\end{array}$ & TFYP (g) \\
\hline \multirow{2}{*}{ TCC (mg/g) } & $\mathrm{P}$ & -0.069 & 0.019 & -0.049 & -0.022 & -0.025 & 0.019 & -0.031 & -0.032 & $0.586 * *$ \\
\hline & $\mathrm{G}$ & -0.160 & 0.045 & -0.116 & -0.052 & -0.059 & 0.045 & -0.071 & -0.075 & $0.586 * *$ \\
\hline \multirow{2}{*}{ CRT (mg/g) } & $\mathrm{P}$ & 0.032 & -0.116 & -0.002 & -0.033 & -0.026 & -0.014 & -0.028 & 0.027 & 0.188 \\
\hline & $\mathrm{G}$ & 0.040 & -0.144 & -0.002 & -0.041 & -0.033 & -0.017 & 0.035 & -0.033 & 0.189 \\
\hline \multirow{2}{*}{ PRC (g/100g) } & $\mathrm{P}$ & 0.162 & 0.004 & 0.223 & 0.095 & 0.129 & -0.036 & 0.109 & 0.143 & $0.748 * *$ \\
\hline & $\mathrm{G}$ & 0.171 & 0.004 & 0.236 & 0.100 & 0.137 & -0.038 & 0.115 & 0.152 & $0.748 * *$ \\
\hline \multirow{2}{*}{$\mathrm{AA}(\mathrm{mg} / \mathbf{1 0 0 g})$} & $\mathrm{P}$ & 0.033 & 0.029 & 0.044 & 0.103 & 0.049 & -0.016 & 0.025 & 0.058 & $0.603 * *$ \\
\hline & $\mathrm{G}$ & 0.023 & 0.020 & 0.030 & 0.071 & 0.034 & -0.011 & 0.017 & 0.041 & $0.603 * *$ \\
\hline \multirow{2}{*}{$\operatorname{MC}(\%)$} & $\mathrm{P}$ & -0.030 & -0.018 & -0.047 & -0.039 & $\underline{-0.082}$ & 0.021 & -0.015 & -0.046 & $0.481 * *$ \\
\hline & $\mathrm{G}$ & -0.013 & -0.008 & -0.019 & -0.016 & -0.034 & 0.009 & -0.006 & -0.019 & $0.484 * *$ \\
\hline \multirow{2}{*}{ OC (mg/100g) } & $\mathrm{P}$ & -0.005 & 0.002 & -0.003 & -0.003 & -0.004 & -0.017 & -0.007 & -0.006 & 0.053 \\
\hline & $\mathrm{G}$ & -0.010 & 0.004 & -0.006 & -0.006 & -0.009 & -0.037 & -0.015 & -0.013 & 0.053 \\
\hline \multirow{2}{*}{ IC (mg/100g) } & $\mathrm{P}$ & -0.073 & 0.039 & -0.080 & -0.039 & -0.030 & 0.068 & -0.165 & -0.086 & 0.262 \\
\hline & $\mathrm{G}$ & -0.063 & 0.034 & -0.069 & -0.034 & -0.026 & 0.059 & -0.142 & -0.075 & 0.262 \\
\hline \multirow{2}{*}{ FA $(\mathrm{mg} / \mathbf{1 0 0 g})$} & $\mathrm{P}$ & 0.027 & 0.013 & 0.037 & 0.033 & 0.033 & -0.021 & 0.040 & $\underline{0.058}$ & $0.571 * *$ \\
\hline & $\mathrm{G}$ & 0.001 & 0.001 & 0.001 & 0.001 & 0.001 & -0.001 & 0.002 & $\overline{0.003}$ & $0.575 * *$ \\
\hline
\end{tabular}

Path using genotypic correlation (Residual effect $=$ SQRT $(1-1.0014)$; phenotypic residual effect $=0.0438$; diagonal underlined values indicate direct effects

$\mathrm{TFYP}=$ total foliage yield per plant, $\mathrm{TCC}=$ total chlorophyll content, $\mathrm{CRT}=$ carotenoids, $\mathrm{PRC}=$ protein content, $\mathrm{AA}=$ ascorbic acid, $\mathrm{MC}=\mathrm{moisture}$ content, $\mathrm{OC}=$ oxalate content, $\mathrm{IC}=$ iron content, $\mathrm{FA}=$ folic acid 
The traits number of branches per plant ($0.262,-0.222,-0.123$ at 30,60 and 90 DAS) and number of leaves per plant (-0.031, 0.122 at 60 and 90 DAS) showed negligible, low and moderate negative direct effect on foliage yield. These results indicated that selection of these traits may be ineffective in improving the yield.

In conclusion, the results of correlation and path coefficient analysis clearly indicate that leaf weight per plant was the most important trait for increasing the yield in vegetable amaranth. Also, leaf/stem ratio, leaf length, leaf width, leaf area index, protein content, ascorbic acid, folic acid and chlorophyll content were important yield components in vegetable amaranth. Thus, the present study suggests that all these traits should be considered simultaneously in selection programme aimed at improving of vegetable amaranth varieties with high yielding (higher yields).

\section{References}

Al Jibouri H A, Miller P A and Robinson H F. 1958. Genotypic and environmental variances and covariances in an upland cotton cross of interspecific origin. Agronomy Journal. 50: 633- 636.

Ahammed A U, Rahman M M and Mian M A K. 2013. Multivariate analysis in stem amaranth. Bangladesh Journal of Plant Breeding and Genetics. 26 (1):11-17.

Aktheruzzaman M. 2013. Morphological characterization of thirty one red amaranth germplasm. M.Sc (Horticulture) thesis submitted to Bangladesh Agricultural University, Mymensingh.

Aruna P. 2009. Correlation and path analysis in amaranthus. The Asian Journal of Horticulture. 4 (2): 361-363.

Arivazhagan and Mohideen, K. 2006. Studies on seed size and seedling in amaranthus
(Amaranthus species). International Journal of Agricultural Sciences. 2: 180-182.

Chattopadhyay A, Das S, Rana P N, Seth T and Dutta S. 2013. Estimation of genetic parameters, inter-relationships and genetic divergence of vegetable amaranths. International Journal of Plant Breeding. 7 (2): 111-115.

Dewey D R and Lu H K. 1959. A correlation and path-coefficient analysis of components of crested wheat grass production. Agronomy Journal. 51: 515518.

Dhangrah V K, Mandal J and Bhat J S. 2015. Heritable variation and predicted selection response on green yield and its component traits in vegetable amaranth. International Journal of Bio-Resource, Environment and Agricultural Sciences (IJBEAS). 1 (4):146-153.

Hasan M, Akther C A and Raihan M S. 2013. Genetic variability, correlation and path analysis in stem amaranth (Amaranthus tricolor L.) genotypes. A Scientific Journal of Krishi Foundation. 11 (1):17.

Gerrano A S, Rensburg W S J V and Adebola $P$ O. 2015. Genetic diversity of amaranthus species in South Africa. South Afrrican Journal of Plant and Soil. 32 (1): 39-46.

Khurana D S, Singh J and Kaur B. 2013. Genetic variability, correlation and path coefficient analysis in amaranthus. Vegetable Science. 40 (2): 238-240.

Mahabub M A R. 2013. Morphological characterization and yield of forty nine stem amaranth germplasm. M.Sc (Horticulture) thesis submitted to Bangladesh Agricultural University, Mymensingh.

National Research Council (1984). Amaranth modern prospects for an ancient crop. National Academy Press.

Raja S, Arjun L, Jaime A, Teixeira D S and 
More T A. 2012. Diversity analysis of fleshy leaf type amaranthus for semiarid ecosystems. International Journal of Plant Breeding. 6 (1):27-33.

Rana, J. C, Yadav, S. K, Mandal, S and Yadav, S. 2005. Genetic divergence and interrelationship analysis in grain amaranth (Amaranthus hypochondriacus L.) germplasm. Indian Journal of Genetics and Plant Breeding. 65 (2): 99-102.

Sarker U, Islam M T, Rabbani M G and Oba S. 2014. Genotypic variability for nutrient, antioxidant, yield and yield contributing traits in vegetable amaranth. Journal of Food, Agriculture \& Environment. 12 (3\&4): 168-174.

Shukla, S. and Singh, S.P. 2002. Genetic divergence in amaranth (Amaranthus hypochondriacus L.). Indian Journal of Genetics and Plant Breeding. 62 (4): 336-337.

Shukla, S., Bhargava, A., Chatterjee, A., Pandey, A.C. and Mishra, B.K. 2010. Diversity in phenotypic and nutritional traits in vegetable amaranth (Amaranthus tricolor L.), a nutritionally underutilized crop. Journal of the Science of Food and Agriculture. 90 (1): 139-144.

\section{How to cite this article:}

Tejaswini, N., K. Ravinder Reddy, P. Saidaiah and Ramesh, T. 2017. Correlation and Path Coefficient Analysis in Vegetable Amaranth (Amaranthus tricolor L.) Genotypes. Int.J.Curr.Microbiol.App.Sci. 6(6): 2977-2996. doi: https://doi.org/10.20546/ijcmas.2017.606.355 